\title{
UNIVERSITY OF MICHIGAN RADIOCARBON DATES XIV
}

\author{
H. R. CRANE and JAMES B. GRIFFIN
}

The University of Michigan, Ann Arbor, Michigan

The following is a list of dates obtained since the compilation of List XIII (R., 1970, v. 12, p. 161-180). The method is essentially the same. Two $\mathrm{CO}_{2}-\mathrm{CS}_{2}$ Geiger counter systems were used. Equipment and counting techniques have been described elsewhere (Crane, 1961). Dates and estimates of error in this list follow the practice recommended by the International Radiocarbon Dating Conferences of 1962 and 1965, in that (a) dates are computed on the basis of the Libby half-life, 5570 yr; (b) A.D. 1950 is used as the zero of the age scale; and (c) the errors quoted are the standard deviations obtained from the number of counts only. In Michigan date lists up to and including VII, we quoted errors at least twice as great as the statistical errors of counting in order to take into account other errors in the over-all process.

We wish to acknowledge the help of Patricia Dahlstrom in preparing chemical samples and John D. Speth, Roberta Pennypacker, Steve Plog, Robert M. Schacht, R. Ingrid Christensen, and Dale D. Brodkey in preparing the descriptions.

\section{GEOLOGIC SAMPLES}

\section{M-2147. Mud Lake site, Michigan}

$$
11,550 \pm 400
$$

9600 B.c.

Calcareous marl from Mud Lake $\left(43^{\circ} 23^{\prime} 30^{\prime \prime} \mathrm{N}\right.$ Lat, $85^{\circ} 40^{\prime} 30^{\prime \prime}$ W Long), Newaygo Co., Michigan. Sample P-56, 730 to $770 \mathrm{~cm}$ below water surface (430 to $470 \mathrm{~cm}$ below sediment surface) tentatively correlated with Valders stadial. Sample from core $3 \mathrm{~m}$ underwater near middle of Mud Lake. Coll. 1968 and subm. by R. O. Kapp, Alma College, Alma, Michigan. Comment (R.O.K.): pollen diagrams show strong oscillation in Picea and Pinus at this level. Oscillation believed to be vegetational response to late glacial warming of Two Creeks Interstadial and subsequent cooling to Valders Stadial. Valders maximum lobe within $50 \mathrm{~km}$ of site. Date corroborates interpretation of late glacial section of Mud Lake pollen diagram. The dated sample is from sediments interpreted to be correlative with the Valders Stadial.

\section{M-2186. Marl Pond, Michigan}

$10,300 \pm 340$

Organic gyttja with intercalated snails $\left(43^{\circ} 28^{\prime} \mathrm{N}\right.$ Lat, $85^{\circ} 42^{\prime} \mathrm{W}$ Long), Newaygo Co., Michigan. Sample from $15 \mathrm{~cm}$ band, lowest of 3 bands of similar dense gyttja, 317.5 to $325 \mathrm{~cm}$ deep. In upper coniferous pollen zone of late glacial time, date should permit correlation with Mud Lake (M-2147) and help stabilize late glacial pollen stratigraphy. Coll. 1968 by R. O. Kapp and Joseph Leach; subm. by R. O. Kapp. Comment (R.O.K.): date corresponds very well with same episode in other pollen diagrams; it is at point at which spruce pollen percentages 
have dropped sharply and pine pollen percentages begin to dominate the record. Dates beginning of postglacial time in W central Michigan.

\section{M-2208. Pittsfield Horse, Michigan}

$330 \pm 100$

Toe bone (Equus) from Pittsfield Twp. $\left(42^{\circ} 14^{\prime} \mathrm{N}\right.$ Lat, $83^{\circ} 45^{\prime} \mathrm{W}$ Long), Washtenaw Co., Michigan. Sample from $\mathrm{N}$ bank of ditch along NE side of Interstate $94, W$ edge of Defiance moraine. Skeleton in depression filled by fine material worked from moraine and surrounding area. Overburden below soil $.761 \mathrm{~m}$. Coll. 1968 by W. R. Farrand and C. W. Hibbard, Univ. of Michigan; subm. by C. W. Hibbard. Comments (C.W.H.): although it looked quite recent, we thought skeleton should be dated since it directly overlay the moraine and since no fossil horse had been reported from Michigan. There was ca. $2.54 \mathrm{~cm}$ zone of burned peat just below soil zone and above bone. (W.R.F.): obviously a horse from historic period.

\section{M-2211. Boney Springs Mammoth site, Missouri 11,750 B.c.}

Organic silt, twigs, seeds, mosses, and other plant debris from tusk cavity of Mammut americanum ( $38^{\circ} 06^{\prime} \mathrm{N}$ Lat, $93^{\circ} 22^{\prime} \mathrm{W}$ Long), Breton Co., Missouri. Coll. 1968 and subm. by Peter Mehringer, Univ. of Arizona, Tucson, Arizona. Comment (P.M.): date agrees with I-3922$16,580 \pm 220$ в.P. (R., 1970, v. 12, p. 90)-in establishing a late Wisconsin age for the bone bed.

\section{Bailer Mammoth site, Michigan}

Bone and carbonized wood fragments from Bailer Mammoth site (43 $40^{\prime} 35^{\prime \prime} \mathrm{N}$ Lat, $84^{\circ} 20^{\prime} 45^{\prime \prime} \mathrm{W}$ Long), Midland Co., Michigan.

\section{M-2145. Bailer Mammoth site}

$24,000 \pm 4000$

Bone of Jefferson Mammoth (id. by Marion Whitney and R. O. Kapp) $2.44 \mathrm{~m}$ below soil surface in basal sandy and gravelly horizon overlain by ca. $1.22 \mathrm{~m}$ organic sediments. Remains were cranial bones, one molar, one fragment of molar, and part of mandible. Assoc. with plant remains, M-2361. Mammoth specimen near $\mathrm{N}$ limits of species. Coll. 1967 and subm. by R. O. Kapp.

\section{M-2361. Bailer Mammoth site}

$$
3310 \pm 160
$$

Cores of Larix laricina, Pinus strobus, and possibly Picea mariana in stratigraphic assoc. with Jefferson Mammoth remains (M-2145).

General Comment (R.O.K.): ancient date of M-2145 indicates that Jefferson Mammoth occupied E Michigan during Mid-Wisconsin interval. Date suggests fossil remains should be assigned to Plum Point Interstadial (late Port Talbot Interstadial, s.l.) described in Ontario by Dreimanis (Goldthwait, et al., 1965). Stratigraphy, alluvial sand and gravel, indicates, and widely disparate dates confirm, that plant remains 
(M-2361) are not synchronous with mammoth bones. This date (M-2361) probably indicates the reworking of Bluff Creek alluvium and burial of bones at recovery point.

\section{ARCHAEOLOGIC SAMPleS \\ A. Upper Mississippi Valley}

White site series, Indiana

Charcoal from White site $\left(40^{\circ} 56^{\prime} \mathrm{N}\right.$ Lat, $85^{\circ} 22^{\prime} 45^{\prime \prime} \mathrm{W}$ Long), T.17N, R.10E, SE $1 / 4$, NW $1 / 4$, Sec. 15, Henry Co., Indiana. Woodland site. Coll. 1967 by John Shrader and Mike Dixon; subm. by B. K. Swartz, Jr., Ball State Univ., Muncie, Indiana.

\section{M-2015. White site}

$$
1860 \pm 200
$$

$$
\text { A.D. } 90
$$

Pit N 22, W 2, $86.4 \mathrm{~cm} \mathrm{~N}$ and $71.1 \mathrm{~cm} \mathrm{E,} 45.7$ to $60.9 \mathrm{~cm}$ deep. Small sample.

\section{M-2016. White site}

$$
1740 \pm 140
$$

Pit N 2, W 3, $43.2 \mathrm{~cm} \mathrm{~N}$ and $127 \mathrm{~cm} \mathrm{E.}$

$$
\text { A.D. } 210
$$

\section{M-2017. White site}

$$
1910 \pm 140
$$

Pit S 1, W 5, $48.3 \mathrm{~cm} \mathrm{~N}$ and $12.7 \mathrm{~cm} \mathrm{E,} 15.2$ to $30.4 \mathrm{~cm}$ deep.

\section{M-2018. White site}

$$
1920 \pm 140
$$

Pit N 2, W 3, $48.3 \mathrm{~cm} \mathrm{~N}$ and $45.7 \mathrm{~cm} \mathrm{E,} 0$ to $15.2 \mathrm{~cm}$ deep.

\section{M-2019. White site}

Pit S 1, W 5, $129.5 \mathrm{~cm} \mathrm{~N}$ and $66 \mathrm{~cm} \mathrm{E,} 15.2$ to $30.4 \mathrm{~cm}$ deep.

\section{M-2020. White site}

Feature 3, pit S 1, W 3, $13.95 \mathrm{~cm}$ deep.

\section{M-2021. White site}

$$
1490 \pm 130
$$

\section{A.D. 460}

$1550 \pm 150$ A.D. 400

$$
1400 \pm 130
$$

A.D. 550

General Comment (B.K.S.): M-2017 and M-2018 from S limit of surviving mound are bark from deposit containing indistinctive Woodland sherds and broken lithic remains. M-2015 is from isolated timber assoc. with burials NW of excavation. M-2016 is from $\log$ tomb assoc. with burials with red ocher, copperwork, expanded bar gorget, pendant, and stone slate celt at $\mathrm{N}$ edge of mound. $\mathrm{M}-2021$ is from log timber in SE portion of mound with no assoc. artifacts. M-2019 and M-2020 may represent an intrusive component in that they are from isolated timbers not assoc. with artifacts. These timbers are centrally located in relation to the defined components. M-2019-2021 seem late for Adena occupation. 
Possibly there was late survival in the $\mathrm{W}$ periphery. In this series, the earliest dates come from the margins of the surviving mound area.

\section{M-2131. Divers site series, Illinois}

$720 \pm 130$

Charcoal from Divers site (MO-28) (Otten site, 11-MO-3, Univ. of

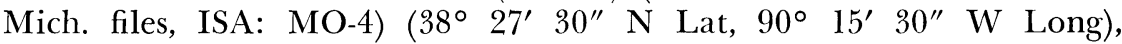
Monroe Co., Illinois. Post in W wall of Feature 15, S 56.9, E 46.57, 56 $\mathrm{cm}$ BAD 124.915. In $1950 \mathrm{~J}$. B. Griffin called site Late Woodland of Pulcher complex. Coll. 1968 and subm. by James Porter, Marshall, Wisc. Comments (J.W.P.): date not out of line; it fits observed sequence of building at site. (J.B.G.): date may fit building sequence, but it is much too late for Pulcher complex.

\section{M-2179. Butcher site, Missouri}

$$
1170 \pm 150
$$

Charcoal from Butcher site (23 C1 118) (39 $27^{\prime} 05^{\prime \prime} \mathrm{N}$ Lat, $94^{\circ} 32^{\prime}$ 10" W Long), Clay Co., Missouri. Sample from Feature 301, shallow, basin-shaped pit. Artifact remains show similarities to materials of SteedKisker site and Doniphan phase of Nebraska tradition. Coll. 1968 by F. A. Calabrese; subm. by W. R. Wood, Univ. of Missouri, Columbia, Missouri. Comment (W.R.W.): date pertains to one of a series of several sites considered a Kansas City variant of Mississippian tradition. Date seems a bit early, but the wide sigma factor precludes any definite assessment at this time.

\section{M-2024. Woodpecker site, Missouri}

$475 \pm 100$

Charcoal from Woodpecker site $\left(37^{\circ} 38^{\prime} 41^{\prime \prime} \mathrm{N}\right.$ Lat, $93^{\circ} 44^{\prime} 23^{\prime \prime}$ W Long), Cedar Co., Missouri. Sample from Sq. 100 NW 90, Feature 63 (pit fill) dug into occupational level. Probable single component, nonceramic site, perhaps Late Archaic. Coll. 1967 by R. E. Pangborn; subm. by W. R. Wood. Comment (W.R.W.): date is later than suggested by cultural remains.

\section{Perrin's Lodge Crematory site series, Illinois}

Wood charcoal from Perrin's Lodge Crematory site $\left(39^{\circ} 20^{\prime} 00^{\prime \prime}\right.$ $\mathrm{N}$ Lat, $90^{\circ} 37^{\prime} 05^{\prime \prime} \mathrm{W}$ Long), Calhoun Co., Illinois. From beneath large limestone slab, obviously in situ and part of crematory structure. Possibility that charcoal is intrusive is remote. In situ fire remnants, Jersey Bluff phase, Late Woodland. Jersey Bluff elbow pipe and sherds in assoc. Coll. 1968 and subm. by Stuart Struever, Northwestern Univ., Evanston, Illinois.

\section{M-2253. Sq. 15, Subsquare 1}

M-2254. Sq. 8, Subsquare 15
$950 \pm 150$ A.D. 1000

$$
1380 \pm 130
$$

A.D. 570

All charcoal from one piece which was at least $7.62 \mathrm{~cm}$ in diam. 
General Comment (S.S.): pottery sherds scattered throughout crematory feature are all "classic" Jersey Bluff ware; expectable dates between A.D. 800 and 1100 . Therefore M-2253 is about expected age; M-2254 is a little earlier than expected from ceramic evidence.

\section{Rodgers Shelter series, Missouri}

Carbonized wood from Rodgers Shelter (23 BE 125) (38 $05^{\prime} 30^{\prime \prime}$ $\mathrm{N}$ Lat, $93^{\circ} 20^{\prime} 40^{\prime \prime} \mathrm{W}$ Long), Benton Co., Missouri. Samples pretreated by C. Vance Haynes, Univ. of Arizona.

\section{M-2281. Rodgers Shelter}

$5200 \pm 200$

Charcoal from a small trash pit, Feature 5418, that originated and terminated in Stratum III, D.D. $1.22 \mathrm{~m}$ to $1.53 \mathrm{~m}$, Sq. $260 \mathrm{NW} 70$, 96.5 to $114.4 \mathrm{~cm} \mathrm{NW} 145 \mathrm{~cm}$ to $152.4 \mathrm{~cm}$. Coll. 1968 by S. A. Ahler; subm. by W. R. Wood. Comment (R.B. McMillan): date is satisfactory since age estimated for Stratum III was 3500 B.P. (top) to 5500 B.P. (bottom), based on cultural materials above and below unit. Stratum III originally thought to be culturally sterile, but small trash pit from which M-2281 came indicates infrequent occupation of shelter as Stratum III was being deposited.

\section{M-2332. Rodgers Shelter}

$5100 \pm 400$

Charcoal from small shallow basin-shaped pit, Feature 5418, containing some refuse within Stratum III; Sq. $260 \mathrm{NW} 70,44-55 \mathrm{NW}$ 76.3 to $119.3 \mathrm{~cm}$; D.D. 1.22 to $1.37 \mathrm{~m}$. Coll. 1968 by S. A. Ahler and R. B. McMillan; subm. by W. R. Wood. Comment (R.B.McM.): during deposition period of Stratum III there was only infrequent occupation. This virtually sterile stratum divided an Early Archaic zone from a later Archaic horizon. Date is satisfactory and generally corroborates date of M-2281 from same feature.

\section{M-2333. Rodgers Shelter}

$10,200 \pm 330$

Carbonized wood from deepest hearth discovered at site, Stratum I, Sq. $225 \mathrm{NW}$ 95, $20.3 \mathrm{~cm}$ NW $43.2 \mathrm{~cm}$, D.D. 9 m. Coll. 1968 by R. B. McMillan and Gerald Berry; subm. by W. R. Wood. Comment (R.B.McM.): cultural debris was scattered around hearth (chert, bone) with no diagnostic artifacts. Hearths just above this location contained Dalton cultural materials; this hearth probably represents initial Dalton occupation. Date is satisfactory.

\section{M-2238. Wright Mound, Kentucky}

$1740 \pm 140$

Wood charcoal (Gleditsia triacantros or Gymnocladus diocica) id. by Suzanne Harris, Univ. of Michigan, from Wright Mound $\left(38^{\circ} 05^{\prime}\right.$ $10^{\prime \prime} \mathrm{N}$ Lat, $83^{\circ} 57^{\prime} 45^{\prime \prime}$ W Long), Montgomery Co., Kentucky (Webb, 1940). Sample from Adena mound Burial 13, Mm6, Feature 19, Sq. 100 
R $8,5.7 \mathrm{~m}$ deep, apparently in secondary mound. Coll. 1939 by J. L. Buckner; subm. by O. C. Shane, Kent State Univ., Kent, Ohio. Comment (O.C.S.): sample dates construction of log tomb intrusive from secondary mound into primary mound. Charred logs covering primary mound dated at $1900 \pm 50$ B.P. by Nuclear Science and Engineering, Inc., Pittsburgh, Pa. (unpub.). Date is consistent with internal stratigraphy of mound and contained cultural material. It is acceptable for late Adena.

\section{M-2239. Dover Mound, Kentucky}

$2260 \pm 140$

Wood charcoal from Dover Mound ( $38^{\circ} 45^{\prime} 20^{\prime \prime} \mathrm{N}$ Lat, $83^{\circ} 53^{\prime} 00^{\prime \prime}$

W Long), Mason Co., Kentucky (Webb and Snow, 1959). Sample from Adena mound Burial 9, Ms 27, from over legs of burial. Coll. 1950 by R. W. Woodbury; subm. by O. C. Shane. Comment (O.C.S.): sample dates bark-covered extended burial in Zone 2 or Zone 3. Sample appears to be stratigraphically older than the 2 previously dated samples, C-759 and C-860 (Science, 1954, v. 119, p. 137), which came from tops of Zone 3 and Zone 4, respectively. C-759 $(2650 \pm 170)$ is suspect, as it is much too early. But C-760, an average of 2 determinations of same sample, is stratigraphically consistent with M-2239. Date is acceptable.

\section{M-2240. Morgan Stone Mound, Kentucky}

$2100 \pm 140$

Bark charcoal, probably Betula Sp., id. by Suzanne Harris from Morgan Stone Mound ( $38^{\circ} 13^{\prime} 30^{\prime \prime} \mathrm{N}$ Lat, $83^{\circ} 51^{\prime} 30^{\prime \prime} \mathrm{W}$ Long), Bath Co., Kentucky (Webb, 1941). Sample assoc. with Burial 6 in Adena mound. Coll. 1939 by Claude Johnston; subm. by O. C. Shane. Comment (O.C.S.): dates initial use of site for burial purposes; structure was burned soon after placement of central burial. Adena Plain and Montgomery Incised vessels grave goods with Burial 6. Date is satisfactory although slightly early for Montgomery Incised pottery, consistently dated to lst century A.D.

\section{M-2241. Hartman Mound, Kentucky}

$2400 \pm 150$

Wood charcoal of Quercus, id. by Suzanne Harris from Hartman Mound (39 $06^{\prime} 00^{\prime \prime} \mathrm{N}$ Lat, $84^{\circ} 49^{\prime} 35^{\prime \prime} \mathrm{W}$ Long), Boone Co., Kentucky. Sample from Burial 7 or 8 of Adena mound (Webb, 1943; Webb and Snow, 1959). Coll. by J. B. Elliot; subm. by O. C. Shane. Comment (O.C.S.): sample from centrally located burial pit over which mound was constructed. This sub-floor pit also contained charred material which, upon analysis, proved to be ". . . some bituminous substance, probably cannel coal” (Webb, 1943, p. 540). While possibility exists that M-2241 was contaminated during combustion, it is important that only Fayette Thick sherds occurred in mound fill, and this date is consistent with other dates for Fayette Thick pottery. Date is acceptable. 
Wood charcoal, (Quercus) id. by Suzanne Harris from Robbins Mound ( $38^{\circ} 54^{\prime} 50^{\prime \prime} \mathrm{N}$ Lat, $84^{\circ} 46^{\prime} 50^{\prime \prime} \mathrm{W}$ Long), Boone Co., Kentucky (Webb and Elliot, 1942). Sample from Feature 26, $24.4 \mathrm{~m}$ to $25.9 \mathrm{~m}$ sec. $\mathrm{E}$ of Curbing and 27.4 to $29 \mathrm{~m}$ sec. of E Curbing, gleaned from unclean cremation on house floor of Adena mound. Coll. 1941 by J. B. Elliot; subm. by O. C. Shane. Comment (O.C.S.): sample obtained from Feature 26 , burned house structure below primary mound at base of accretional Robbins tumulus. Dates initial stage of mound construction and Adena Plain sherds occurring in the ash deposits of Feature 26. Date is acceptable.

Miller site series, Indiana

$5220 \pm 200$

3270 B.c.

Charcoal, both wood and hickory nut, from Miller site $\left(38^{\circ} 01^{\prime}\right.$ $00^{\prime \prime} \mathrm{N}$ Lat $86^{\circ} 11^{\prime} \mathrm{W}$ Long), Mauckport, Ind.-Ky. quad., Sec. 3, T.6S, R.3E, NW quarter, to $\mathrm{W}$ of Buck Creek, Harrison Co., Indiana. Midden from which samples were taken was a distinctive zone resting on a stratum of mussel shells. Samples combined. Coll. 1970 and subm. by D. E. Janzen, Beloit College, Beloit, Wisconsin.

\section{M-2389. Miller site}

Test Pit A, 15.2 to $22.8 \mathrm{~cm}$ below datum, from midden area; no assoc. features.

M-2390. Miller site

Test Pit A, 22.8 to $30.4 \mathrm{~cm}$ below datum, from midden; no assoc. features.

\section{M-2391. Miller site}

Test Pit A, 30.4 to $38.1 \mathrm{~cm}$ below datum, from midden area; no assoc. features.

General Comment (D.E.J.): date based on 3 samples ranging from 15.2 to $38.1 \mathrm{~cm}$ at Miller site and is composite date from 3 levels. It represents an Archaic horizon and fits with dates from the Hornung site, Kentucky (M-2460: $4900 \pm 200 ;$ M-2461: $5220 \pm 230 ;$ M-2464: $5000 \pm 200$ ).

\section{Hornung site series, Kentucky}

Charred nuts from Hornung site $\left(38^{\circ} 00^{\prime} 00^{\prime \prime} \mathrm{N}\right.$ Lat, $85^{\circ} 56^{\prime} 15^{\prime \prime}$ W Long), USGS Fort Knox, Kentucky, knoll N of confluence of Pond Creek and Salt R. Coll. 1970 and subm. by D. E. Janzen.

\section{M-2460. Hornung site}

$4900 \pm 200$

Charred nuts, possibly hickory, from Unit 3, Level 10, from midden.

M-2461. Hornung site

$5220 \pm 230$

3270 B.c.

Charred nuts, possibly hickory, from Unit 3, Level 13. Small sample. 
M-2464. Hornung site

Unit 8, Level 4.

General Comment (D.E.J.): M-2460 and M-2461 date earliest Archaic horizons at Hornung site. Dates agree with expectations. M-2464 should date Early Woodland component; 3050 B.c. is erroneous.

\section{Riverwood Rock Shelter, Kentucky}

Charred wood and nuts from Riverwood Rock Shelter $\left(38^{\circ} 00^{\prime} 20^{\prime \prime}\right.$ $\mathrm{N}$ Lat, $85^{\circ} 40^{\prime} 30^{\prime \prime}$ W Long), USGS Brooks, Kentucky, on $\mathrm{N}$ bank of Salt R. Coll. 1970 and subm. by D. E. Janzen.

\section{M-2462. Riverwood Rock Shelter}

$2870 \pm 150$

Charred wood and nuts, hickory definitely present, from 495 E 545, Level 5.

\section{M-2463. Riverwood Rock Shelter}

Charred nuts, hickory definitely present, from $500 \mathrm{E} 590$, Feature 3, a small pit.

General Comment (D.E.J.): M-2462 and M-2463 date Early Woodland component. Assoc. ceramics were thick grit-tempered ware characteristic of Early Woodland.

\section{B. Great Lakes}

\section{Indian Hills site series, Ohio}

Charred wood from Indian Hills site $\left(41^{\circ} 36^{\prime} \mathrm{N}\right.$ Lat, $83^{\circ} 32^{\prime} \mathrm{W}$ Long), Wood Co., Ohio. Coll. 1967 and subm. by E. J. Prahl, Univ. of Toledo, Toledo, Ohio.

M-2042. Indian Hills site A.D. 1110

$840 \pm 100$

T.P. O-E-3, SE $1 / 4, .275 \mathrm{~m}$ deep. Dates pottery at this level.

M-2043. Indian Hills site T.P. O-E-2, .489 to $.55 \mathrm{~m}$ deep.

M-2044. Indian Hills site

C Doze area. House No. 1, Burial 1, Trench A, .702 to $.794 \mathrm{~m}$ deep. Dates house.

\section{M-2268. Indian Hills site} A.D. 1610

$$
340 \pm 100
$$

Charcoal from Pit 6, Feature 1, 1.28 to $1.31 \mathrm{~m}$ level. Dates ceramics from pit.

General Comment (E.J.P.): dates for M-2042 to M-2044 seem too early for the shell-tempered pottery and other artifacts; M-2268 seems too late. 


\section{M-2087. Morrin site, Michigan}

A.D. 1070

$880 \pm 110$

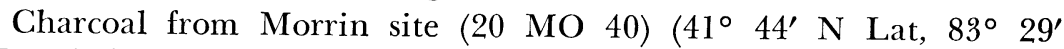
W Long), Monroe Co., Michigan. Sample from Test Pit 1, NW $1 / 4$ of $.61 \mathrm{~m}$ floor, assoc. with Glen Meyer-like pottery of Ontario (Wright, 1966). Coll. 1967 and subm. by E. J. Prahl. Comment (E.J.P.): good date for material recovered. Ceramics appear similar to Glen Meyer focus ceramics.

\section{M-2310. Gladieux site, Ohio}

Charcoal from Gladieux site (33 Lu 7) $\left(41^{\circ} 35^{\prime} 45^{\prime \prime} \mathrm{N}\right.$ Lat, $83^{\circ} 36^{\prime}$ 00" W Long), Adams Twp., Sec. 28, Lucas Co., Ohio, from Test Pit 1, Feature 1. Dates ceramics assoc. with Feature 1, firepit. Coll. 1969 and subm. by E. J. Prahl. Comment (E.J.P.): lithic artifacts are Middle Woodland. Date is too late.

\section{Ekdahl-Goudreau site series, Michigan}

Charcoal from Ekdahl-Goudreau site $\left(45^{\circ} 55^{\prime} \mathrm{N}\right.$ Lat, $85^{\circ} 55^{\prime} \mathrm{W}$ Long), Sec. 21, Mueller Twp. (T41N-R13W), Schoolcraft Co., Michigan. Coll. 1968 and subm. by E. J. Prahl.

\section{M-2311. Ekdahl-Goudreau site}

$$
870 \pm 120
$$

Test Pit O-SW, NE 1/4, S. S. 4-5 .24 m. Dates Late Woodland level. Comment (E.J.P.): dates top level of this 2-component site and is assoc. with Juntunen-like ceramics. Would compare favorably with A.D. 1060 \pm 75 date from Juntunen dating Bois Blanc ware (M-1140: R., 1961, v. 3, p. 110).

\section{M-2312. Ekdahl-Goudreau site}

$$
1290 \pm 130
$$

Test Pit O-SW, NE 1/4, S. S. 9-10, .55 to $.58 \mathrm{~m}$. Dates Laurel level of site. Comment (E.J.P.): should date Middle Woodland ceramics from base. Date is late, compared to Middle Woodland dates by Brose at Summer I. on somewhat comparable material (R., 1970, v. 12, p. 171-172).

\section{M-2313. Cufr site, Ohio}

$1120 \pm 120$

Charcoal from Cufr site (33 FU 2) (41 $37^{\prime} 34^{\prime \prime} \mathrm{N}$ Lat, $83^{\circ} 55^{\prime} 29^{\prime \prime}$ W Long), Sec. 10, Fulton Twp. (R4E-T9S), Fulton Co., Ohio. Pit 12 .52 to $.61 \mathrm{~m}$ level. Coll. and subm. by E. J. Prahl. Comment (E.J.P.): dates Late Woodland ceramics with high collar and fabric-impressed surface. Date is early for this material within Younge tradition but consonant with Wayne ware found elsewhere in site.

\section{Allegan Dam site series, Michigan}

Charcoal from Allegan Dam site $\left(42^{\circ} 34^{\prime} \mathrm{N}\right.$ Lat, $85^{\circ} 57^{\prime} 30^{\prime \prime} \mathrm{W}$ Long), Allegan Co., Michigan. Site is Late Woodland camp with many 
storage pits containing grit- and shell-tempered ceramics. Coll. 1968 and subm. by E. E. Baldwin, Western Michigan Univ., Kalamazoo, Michigan.

M-2230. Allegan Dam site

$740 \pm 100$ fire pit.

Feature 19, Level 3. Material dated is charcoal from stone-lined

\section{M-2231. Allegan Dam site}

$640 \pm 100$

Feature 17, 60.9 to $89 \mathrm{~cm}$ below surface. Sample is burned wood from a storage pit.

General Comment (E.E.B.): dates are acceptable for shell tempering in SW Michigan and suggest that this is a single component site.

\section{6th Street site series, Michigan}

Charcoal and charred wood from 46 th Street site $\left(42^{\circ} 37^{\prime} \mathrm{N}\right.$ Lat, $86^{\circ} 00^{\prime}$ W Long), Allegan Co., Michigan, a Late Woodland camp site. Ceramics are grit-tempered, with stylistic relationship to Wayne ware as described at Michigan sites of Riviere au Vase and Spring Creek. Coll. 1968 and subm. by E. E. Baldwin.

\section{M-2232. 46th Street site}

Charcoal from Feature 4, Level 5, hearth.

\section{M-2233. 46th Street site}

Charred wood from a storage pit, Feature $6,104.3$ to $137 \mathrm{~cm}$ below surface.

\section{M-2236. 46th Street site}

A.D. 1710

A.D. 1140

$$
910 \pm 100
$$

Burned wood from burial pit in Mound 1. Burial removed by previous excavator (unknown).

General Comment (E.E.B.): M-2232 and M-2233 are in expected time range. M-2236 is surprisingly late, perhaps due to disturbance. Relationship of this and several similar mounds to adjacent Late Woodland village site (M-2232, M-2233) remains problematic.

\section{Nordhof site series, Michigan}

Charcoal and burned wood from Nordhof site (20 Al 13) $\left(42^{\circ} 38^{\prime}\right.$ $30^{\prime \prime} \mathrm{N}$ Lat, $86^{\circ} 07^{\prime} 30^{\prime \prime} \mathrm{W}$ Long), Allegan Co., Michigan. Coll. 1968 and subm. by E. E. Baldwin.

\section{M-2234. Nordhof site}

$$
\begin{aligned}
910 & \pm 110 \\
\text { A.D. } 1040 &
\end{aligned}
$$

Charcoal from small fire pit, Feature $26,50.8$ to $73.7 \mathrm{~cm}$ below surface. Shell-tempered pottery and some grit-tempered ware overlay feature, presumably assoc. Comment (E.E.B.): date is somewhat early 
but is in acceptable range for appearance of shell-tempered pottery in SW Michigan.

\section{M-2235. Nordhof site}

Burned wood from storage pit, Feature 25, 66 to $76.3 \mathrm{~cm}$ below surface. Comment (E.E.B.): this date and M-2234 confirm suspected multiple component nature of this extensive site.

\section{M-2237. Brainerd site, Michigan}

$1510 \pm 130$

Human bone from Brainerd site $\left(42^{\circ} 32^{\prime} 30^{\prime \prime} \mathrm{N}\right.$ Lat, $85^{\circ} 58^{\prime} \mathrm{W}$ Long), Allegan Co., Michigan. Trench A, 73.7 to $152.4 \mathrm{~cm}$ below surface. No cultural remains assoc. Ossuary, low mound with remains of $>30$ individuals. Coll. 1968 and subm. by E. E. Baldwin. Comment (E.E.B.): skeletal remains entirely disarticulated and very fragmentary due, in part, to prior disturbance.

\section{Holtz site series, Michigan}

Charcoal from Holtz site (44 $58^{\prime} 45^{\prime \prime} \mathrm{N}$ Lat, $85^{\circ} 11^{\prime} 23^{\prime \prime} \mathrm{W}$ Long), Antrim Co., Michigan. Coll. 1967 and subm. by C. E. Cleland, Michigan State Univ. Mus., East Lansing, Michigan.

\section{M-2061. Holtz site}

\section{A.D. $1380 \mathrm{~N}$}

Charcoal from Unit S20 W10, between 0 and $.092 \mathrm{~m}$ deep, assoc. with pottery.

\section{M-2062. Holtz site}

$$
770 \pm 100
$$

\section{A.D. 1180}

A.D. $1210 \mathrm{~N}$

Charcoal from Unit S20 W10, Level 2, .76 to $1.02 \mathrm{~cm}$ depth. General Comment (G.E.C.): Holtz site is late Middle Woodland occupation in zone covered by charred peat. Samples M-2061 and M-2062 do not date occupation but may correspond to surface burnings after occupation.

\section{Wycamp Creek site series, Michigan}

Charcoal from Wycamp Creek site $\left(45^{\circ} 39^{\prime} \mathrm{N}\right.$ Lat, $85^{\circ} 00^{\prime} 45^{\prime \prime} \mathrm{W}$ Long), Emmet Co., Michigan. Coll. 1967 and subm. by C. E. Cleland.

\section{M-2059. Wycamp Creek site}

Charcoal from hearth, Feature 2, from S 1/2 of Unit N30 E190, at depth .366 to $.458 \mathrm{~m}$ below surface.

M-2060. Wycamp Creek site

$$
240 \pm 100
$$

Charcoal from Unit Test 2, at depth $.825 \mathrm{~m}$ below surface. 


\section{M-2065. Wycamp Creek site}

Charcoal seeds from depth $.275 \mathrm{~m}$ in Unit $\mathrm{S} 150 \mathrm{E} 40$, assoc. with a flint concentration.

General Comment (C.E.C.): this series indicates occupation from late Middle Woodland to Early Historic periods. M-2065 may be slightly late for Laurel occupation of Wycamp beach. M-2059 probably dates Bois Blanc phase occupation of Late Woodland component, while M-2060 dates ceramics thought to represent an historic Ottawa occupation.

\section{M-2063. Henderson-Lamb site, Michigan}

$500 \pm 100$

A.D. 1450

A.D. $1420 \mathrm{~N}$

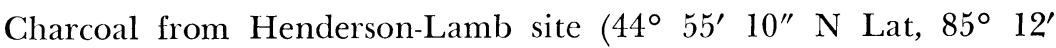
$20^{\prime \prime}$ W Long), Antrim Co., Michigan. Sample from Level 3, .183 to .275 $\mathrm{m}$ below surface, of Unit N80 E60. Coll. 1967 and subm. by C. E. Cleland. Comment (C.E.C.): similarities in ceramic styles from Skegemog Point site, Fauver site, and Henderson-Lamb site indicate similar temporal placements for these occupations. Fauver site date A.D. $1495 \pm 100$ (R., 1968, v. 10, p. 83) and Skegemog Point site date A.D. $1310 \pm 110$ (ibid.) agree with A.D. $1420 \pm 100$ date for Henderson-Lamb and this is probably accurate for this occupation.

\section{O'Neill site series, Michigan}

Charcoal from O'Neill site (45 $15^{\circ} 7^{\prime} \mathrm{N}$ Lat, $85^{\circ} 21^{\prime} \mathrm{W}$ Long), NE $1 / 4$ Sec. 1, T.33N R.9W, NW $1 / 4$ of SW $1 / 4$ of T.33N R.8W, Charlevoix, Emmet Co., Michigan. Coll. 1969 and subm. by C. E. Cleland.

\section{M-2398. O'Neill site}

$$
430 \pm 100
$$

A.D. 1520

A.D. $1455 \mathrm{~N}$

Charcoal from hearth in 3rd level $.275 \mathrm{~m}$ of Unit N350 W40.

\section{M-2401. O'Neill site}

Charcoal from hearth in N340 W40 (small sample).
$1000 \pm 140$

\section{A.D. 950}

A.D. $1000 \mathrm{~N}$

M-2405. O'Neill site

A.D. 1280

A.D. $1290 \mathrm{~N}$

Charcoal from hearth at $.732 \mathrm{~m}$ below surface, Unit N380 W50.

M-2406. O'Neill site
$740 \pm 100$

A.D. 1210

A.D. $1210 \mathrm{~N}$

Charcoal from hearth in Occupation Zone 2, ca. .671 m below surface, in Unit N390 W60. 
General Comment (C.E.C.): this series indicates site was occupied from ca. A.D. 1000 to 1500 . M-2398 probably dates a late Juntunen phase house. M-2405 and M-2406 date an early Juntunen phase occupation. M-2401 might possibly date a Skegemog occupation.

\section{Eagle Island site series, Michigan}

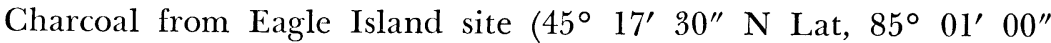
W Long), SE 1/4 of SE 1/4, Sec. 34, T.34N R.6W, Walloon Lake, Emmet Co., Michigan. Coll. 1969 and subm. by C. E. Cleland.

\section{M-2400. Eagle Island site}

$2270 \pm 140$

Charcoal from hearth, from SW corner of Level 4 in Unit N30 E50.

\section{M-2404. Eagle Island site}

Charcoal from hearth, Feature 3, Units N30 E40 and N30 E50.

General Comment (C.E.C.): both samples are clearly too early to date occupation of Eagle Island site. Ceramics from site indicate a Late Woodland temporal placement.

\section{M-2402. North Manitou \#3 site, Michigan}

$\mathbf{3 0 3 0} \pm \mathbf{2 0 0}$

Charcoal from North Manitou \#3 site $\left(45^{\circ} 08^{\prime} \mathrm{N}\right.$ Lat, $85^{\circ} 58^{\prime} \mathrm{W}$ Long), NE 1/4 of SE 1/4, Sec. 27, T.32N R.14W, North Manitou I., Leelanau Co., Michigan. Charcoal from hearth in the $\mathrm{N} \mathrm{1/2}$ of Test No. 1, Level 2; small sample. Coll. 1968 and subm. by C. E. Cleland. Comment (C.E.C.): site is on a Nipissing beach terrace with Late Woodland and Archaic occupations. M-2402 seems to date Archaic component.

\section{M-2403. Scott site, Michigan}

\section{A.D. 1000 \\ A.D. $1060 \mathrm{~N}$}

$950 \pm 110$

Charcoal from Scott site (43 $56^{\prime} 15^{\prime \prime} \mathrm{N}$ Lat, $86^{\circ} 15^{\prime} 38^{\prime \prime}$ W Long), NE $1 / 4$ of SE 1/4, Sec. 19, and NW 1/4 of SW 1/4, Sec. 20, Custer Twp., Ludington, Mason Co., Michigan. Charcoal from hearth, S1 E1-S3 E1, Pit H, St. I-IV. Coll. 1969 and subm. by C. E. Cleland. Comment (G.E.C.): dates Late Woodland component.

\section{Ponshewaing Point site series, Michigan}

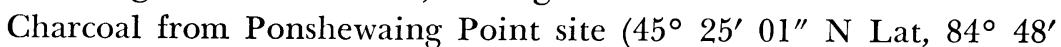
$00^{\prime \prime}$ W Long), SW 1/4 of SE 1/4, Sec. 16, Littlefield Twp., Emmet Co., Michigan.

\section{M-2064. Ponshewaing Point site} $1760 \pm 130$

Charcoal from hearth, Feature 1 in Unit S140 W60. Coll. 1967 and subm. by C. E. Cleland. 


\section{M-2399. Ponshewaing Point site}

\section{Cleland.}

Charcoal from Level 4 of S370 EO. Coll. 1969 and subm. by C. E.

General Comment (C.E.C.): site is a Late Woodland occupation with ceramics indicating time span between A.D. 1000 and 1400. M-2399 and M-2064 do not date occupation.

\section{Wood site series, Michigan}

Charcoal from Wood site $\left(45^{\circ} 22^{\prime} 12^{\prime \prime} \mathrm{N}\right.$ Lat, $85^{\circ} 07^{\prime} 00^{\prime \prime} \mathrm{W}$ Long), Charlevoix Co., Michigan. Coll. 1967 and subm. by C. E. Cleland.

M-2057. Wood site

$990 \pm 120$

$$
\text { A.D. } 960
$$

A.D. $1020 \mathrm{~N}$

Charcoal from black area, with pottery, Level 4 of NE $1 / 4$ of N65 W85, along $\mathrm{N}$ wall.

\section{M-2058. Wood site}

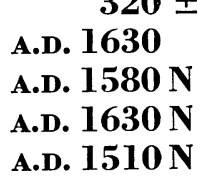

Charcoal from hearth in Level 4 of Unit S10 E140.

General Comment (C.E.C.): normalized dates for M-2058 provide 3 alternatives of which the earliest, A.D. $1510 \pm 100$, is the preferred choice to date castellated cord-impressed Juntunen-type pottery. Date agrees with M-2398 from O'Neill site which dates a late Juntunen-phase house. M-2057 supposedly dates earlier Juntunen pottery with stab-drag impressions; A.D. $1020 \pm 120$ seems too early for wares of this variety.

\section{Fort Michilimackinac series, Michigan}

Charcoal from Fort Michilimackinac $\left(45^{\circ} 49^{\prime} 50^{\prime \prime} \mathrm{N}\right.$ Lat, 84 $44^{\circ}$ $50^{\prime \prime}$ W Long), Mackinaw City, Emmet Co., Michigan. Middle Woodland component. Samples from Feature 169, Laurel tradition fire pit. Laurel tradition indicated by pottery and flint tool assoc. Coll. 1970 and subm. by L. M. Stone, The Museum, Michigan State Univ., East Lansing, Michigan.

\section{M-2489. Sample MS ${ }^{2}$-5388-1}

$$
1500 \pm 120
$$

Sample possibly contaminated with recent charcoal. Comment (L.M.S.): date is consistent with field interpretation and comparative dating of Late Laurel tradition sites.

\section{M-2490. Sample MS $^{2}-5388-2$}

$$
1300 \pm 130
$$

Comment (L.M.S.): date of Laurel component is acceptable but between 100 and $200 \mathrm{yr}$ later than anticipated in reviewing previously dated sites. 


\section{Northeastern United States}

\section{Kinzua site series, Pennsylvania}

Wood charcoal from Kinzua site (36 Wa 53) (41 $53^{\prime} 06^{\prime \prime} \mathrm{N}$ Lat, $78^{\circ} 57^{\prime} 05^{\prime \prime}$ W Long), Warren Co., Pennsylvania. Samples from inside rectangular Proto-Iroquoian house. Coll. 1965 and subm. by D. W. Dragoo, Carnegie Mus., Pittsburgh, Pa.

M-2192. Kinzua site F. C. 4856

$750 \pm 100$

From small, circular fire pit inside house.

A.D. 1200

M-2193. Kinzua site F. C. 4866

A.D. 1350

$600 \pm 100$

From large storage pit inside house.

General Comment (D.W.D.): M-2192 appears reasonably close to early occupation of site. Ceramics would conform to this time period in early Proto-Iroquoian. M-2193 seems a little late; it should be similar to M-2192.

\section{Cornplanter site series, Pennsylvania}

Wood charcoal from Cornplanter site (36 Wa 83) $\left(41^{\circ} 55^{\prime} 36^{\prime \prime} \mathrm{N}\right.$ Lat, $78^{\circ} 55^{\prime} 59^{\prime \prime}$ W Long), Warren Co., Pennsylvania.

\section{M-2194. Cornplanter site, Grant No. 1}

$$
970 \pm 100
$$

A.D. 980

Sample from fire hearth in midden zone, 53.4 to $60.9 \mathrm{~cm}$ below surface from Early Proto-Iroquoian site. Coll. 1965 and subm. by D. W. Dragoo.

\section{M-2195. Cornplanter site, Grant No. 2}

Sample from refuse pit containing transitional or Late Archaic material. Coll. 1966 by Stanley Lantz; subm. by D. W. Dragoo.

General Comment (D.W.D.): M-2194 is extremely close to our estimate of A.D. 1000 for this early Proto-Iroquoian site. M-2195 is reasonable for Proto-Iroquoian occupation but does not date Archaic items in feature, which may have been brought by Proto-Iroquoian peoples.

\section{Onoville Bridge site series, New York}

Wood charcoal from Onoville Bridge site (30 Ca 5) $\left(42^{\circ} 01^{\prime} 40^{\prime \prime}\right.$ $\mathrm{N}$ Lat, $78^{\circ} 56^{\prime} 55^{\prime \prime} \mathrm{W}$ Long), Cattaraugus Co., New York. Coll. 1966 and subm. by D. W. Dragoo.

M-2196. Onoville site, F. C. 5292

$$
180 \pm 100
$$

From W end of trench surrounding stockaded Proto-Iroquoian village site.

M-2197. Onoville site, F. C. 5295

$$
520 \pm 100
$$

From Feature 103, S portion of trench surrounding village. 
General Comment (D.W.D.): M-2196 is completely out of line with contents of feature. On basis of pottery types, site was estimated at ca. A.D. 1400. M-2197 is close to this estimate.

\section{M-2198. Drew site, Pennsylvania}

$$
590 \pm 100
$$

\section{A.D. 1360}

Charred corn kernels from Drew site (36 Al 62) (40 22' $04^{\prime \prime} \mathrm{N}$ Lat, $80^{\circ} 07^{\prime} 08^{\prime \prime} \mathrm{W}$ Long), Alleghany Co., Pennsylvania. From large, irregular refuse pit of Late Prehistoric Monongahela site. Coll. 1965 by William Buker; subm. by D. W. Dragoo. Comment (D.W.D.): date seems a little late for an early Monongahela site.

\section{M-2200. Bunola site, Pennsylvania}

$$
600 \pm 100
$$

Charcoal from Bunola site (36 Al 4) $\left(40^{\circ} 14^{\prime} 12^{\prime \prime} \mathrm{N}\right.$ Lat, $79^{\circ} 57^{\prime}$ $36^{\prime \prime}$ W Long), Alleghany Co., Pennsylvania. Sample from Feature 1, large but shallow basin-shaped pit of Late Prehistoric village. Coll. 1966 by D. P. Tanner; subm. by D. W. Dragoo. Comment (D.W.D.): date seems quite good for this Monongahela site.

\section{M-2201. McKees Rocks site, Pennsylvania}

\section{A.D. 1330}

$620 \pm 100$

Charcoal from McKees Rock site (36 Al 16) $\left(40^{\circ} 28^{\prime} 20^{\prime \prime} \mathrm{N}\right.$ Lat, $80^{\circ}$ 02' 59" W Long), Alleghany Co., Pennsylvania. Charcoal from Feature 39, pit in Late Prehistoric Monongahela site. Coll. 1960 by William Buker; subm. by D. W. Dragoo. Comment (D.W.D.): date is close to our estimate based on pottery types at this site.

\section{M-2202. Anderson Mound, Pennsylvania}

$390 \pm 100$

Charcoal from Anderson Mound (36 Al 96) $\left(40^{\circ} 30^{\prime} 56^{\prime \prime} \mathrm{N}\right.$ Lat, $79^{\circ} 50^{\prime} 49^{\prime \prime}$ W Long), Alleghany Co., Pennsylvania. Wood charcoal assoc. with Burial No. 1, cremated burial in Middle Woodland stone mound. Coll. 1964 by R. L. George; subm. by D. W. Dragoo. Comment (D.W.D.): sample cannot be considered Middle Woodland mound; it must have been contaminated or was recent, since mound was disturbed in historic times. Sample appeared undisturbed.

\section{M-2203. Portman site, Pennsylvania}

Wood charcoal from Portman site (36 Al 39) (40 19' 06" N Lat, $80^{\circ} 06^{\prime} 47^{\prime \prime} \mathrm{W}$ Long), Alleghany Co., Pennsylvania. From base of Feature 2, medium-sized fire pit from Late Prehistoric village site. Coll. by D. P. Tanner; subm. by D. W. Dragoo. Comment (D.W.D.): date is close to expected for this early Monongahela site.

\section{M-2204. Campbell site, Pennsylvania}

Wood charcoal from Campbell site (36 Fa 26) $\left(40^{\circ} 00^{\prime} 32^{\prime \prime}\right.$ N Lat, $79^{\circ} 51^{\prime} 04^{\prime \prime}$ W Long), Fayette Co., Pennsylvania. Sample from Feature 1, refuse pit of Late Prehistoric Monongahela site. Coll. 1965 and subm. 
by D. W. Dragoo. Comment (D.W.D.): date appears good for this Middle Monongahela site.

\section{M-2205. Hartly site, Pennsylvania}

$250 \pm 100$

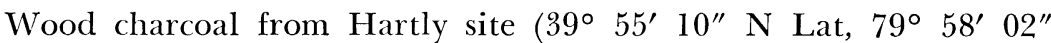

W Long), Green Co., Pennsylvania. Sample from Feature 6, refuse pit of Late Prehistoric Monongahela (Early phase) site. Coll. 1964 and subm. by D. W. Dragoo. Comment (D.W.D.): pottery types invalidate date for site, which is early Monongahela. Date should be close to A.D. 1360 .

\section{Chambers Mound series, Pennsylvania}

Wood charcoal from Chambers Mound (36 Lr 11) $\left(41^{\circ} 00^{\prime} 48^{\prime \prime}\right.$ N Lat, $80^{\circ} 25^{\prime}$ 52" W Long), Lawrence Co., Pennsylvania. Coll. 1960 by John Zakucia; subm. by D. W. Dragoo.

\section{M-2206. Chambers Mound F. C. 3674}

$120 \pm 100$

Wood charcoal from fill of Middle Woodland stone mound.

M-2207. Chambers Mound F. C. 3729

$3070 \pm 150$

1120 B.C. mound.

Wood charcoal from small fire pit in transitional zone beneath

General Comment (D.W.D.): M-2206 is unsatisfactory. Since this Early Woodland-Middle Woodland mound was disturbed in historic times, it is possible that charcoal came from a disturbed zone. Sample should have been close to 1120 в.c. M-2207 date seems good for Transitional Archaic zone under Chambers Mound.

\section{M-2247. Cornplanter Grant No. 3 site, M-2248. Pennsylvania \\ $240 \pm 100$}

Wood charcoal from Cornplanter Grant No. 3 site (36 Wa 81) $\left(41^{\circ}\right.$ $58^{\prime} 10^{\prime \prime} \mathrm{N}$ Lat, $78^{\circ} 55^{\prime} 50^{\prime \prime} \mathrm{W}$ Long), Warren Co., Pennsylvania. Sample from upper and lower levels of large oval-shaped cremation pit containing 3 large "turkey tail" blades and tubular pipe fragments. Samples combined. Coll. 1966 by Stanley Lantz; subm. by D. W. Dragoo. Comment (D.W.D.): this date for Red Ocher culture feature does not seem to fit with any previous dates for period. It seems ca. $2500 \mathrm{yr}$ too late.

\section{M-2255. Harlan Mill steatite quarry, Maryland}

$3330 \pm 160$

1380 B.C.

Twigs of charcoal (Populus, maybe Salix 9, Juglans 3, Quercus 4, Fraxinus 5, and Picea 1) id. by Suzanne Harris, from Harlan Mill steatite quarry (39 $39^{\prime} 38^{\prime \prime} \mathrm{N}$ Lat, $75^{\circ} 52^{\prime} 40^{\prime \prime} \mathrm{W}$ Long), Cecil Co., Maryland. Sample from hearth in $\mathrm{E}$ end of excavation, approx. $1.37 \mathrm{~m}$ below ground surface (Wilkins, 1962, p. 1-22). Coll. and subm. by E. S. Wilkins, Jr., R 2, Newark, Delaware. Comment (E.S.W.): date indicates quarry- 
ing of material for carved stone bowls during Transitional stage at horizon of Perkiomen and Orient points.

\section{Lower Mississippi Valley and Southeastern U.S.}

\section{M-1968. Dupree site, Arkansas}

$410 \pm 100$

Charcoal from Dupree site (3 PH 1) (34 $11^{\prime} \mathrm{N}$ Lat, $91^{\circ} 00^{\prime} \mathrm{W}$ Long), NE 1/4, SW 1/4, Sec. 10, Twp. 65, Rge. 1 E, Phillips Co., Arkansas. From base of Level 2, approx. $30.4 \mathrm{~cm}$ deep, from refuse cover assoc. with shell-tempered pottery, Wallace Incised (Moselage, 1965). Coll. 1967 and subm. by John Moselage, Memphis, Tennessee. Comment (J.M.): pottery from this site is being considered as possibly made by Quapaw people; date fits with site and is reasonable for historical date of 1673. Sample was from a lower level; possibly from early phase of development of Quapaw tribe which eventually became historical. Site was occupied for a considerable period of time.

\section{M-2088. Beckwith's Fort site, Missouri}

A.D. 1530

$420 \pm 100$

Wood charcoal from Beckwith's Fort site $\left(36^{\circ} 43^{\prime} \mathrm{N}\right.$ Lat, $89^{\circ} 15^{\prime}$ W Long), Mississippi Co., Missouri. Sample from floor of burned house (House I, 23 MI 2), Sq. 2480 R 2330, AE 99.6, at basal .153 m of house fill. Directly assoc. with vessels of Mississippian form, but grog and grogshell tempering, with paste like Baytown ware. Date will place ceramic style in area as well as fortified village-ceremonial center (Smith, 1967; Thomas, 1894; Williams, 1954). Coll. 1967 by J. W. Clark; subm. by G. P. Smith, Univ. of Missouri. Comment (G.P.S.): at first, date seemed nearly 200 yr too late. Ref. to Table 1 in Stuiver and Suess (1966, p. 537) suggests calendar equivalent of A.D. $1460 \pm 100$. Actual date is probably in lst half of indicated range. The Bryant phase is currently considered to mark the ceramic transition from grog-tempered Beckwith phase ceramics to shell-tempered ceramics of the Cairo Lowland phase. Date emphasizes contrast between ceramic tradition of $\mathrm{N}$ end of Mississippi Alluvial Valley and those to $\mathrm{S}$ where shift to shell tempering occurred much earlier.

\section{Hyneman site series, Arkansas}

Charred nut shell and wood from Hyneman site (3 PO 54) $\left(35^{\circ}\right.$ 03' 33" N Lat, 90 34' 30" W Long), Poinsett Co., Arkansas. Should date early part of Hyneman phase, ca. A.D. 900 to 1100. Coll. 1967 and subm. by D. F. Morse, State Univ., Arkansas.

\section{M-2112. Hyneman site}

Feature 6, refuse pit, charred hickory nut shell.

M-2113. Hyneman site

$$
1330 \pm 130
$$

$$
1210 \pm 130
$$

A.D. 740 
General Comment (D.F.M.): dates are acceptable; they place Late Woodland (Baytown) assemblage shortly before change to Mississippian.

M-2212.

M-2213. Hoecake site, Missouri

$1310 \pm 130$

A.D. 640

Wood charcoal from Hoecake site (23 Mi 8) $\left(36^{\circ} 45^{\prime} \mathrm{N}\right.$ Lat, $89^{\circ} 15^{\prime}$

W Long), Mississippi Co., Missouri. From partially charred $\log$ in tombs of Story Mound, subsurface, log-lined tomb (1 of 3) below large, conical mound. No direct assoc. with burials in tomb, but some un. intentional debris was found in tomb. Assoc. with Mulberry Creek Cord-Marked, Baytown Plain, clay- and shell-tempered sherds, and a shell-tempered loop handle. Will date tombs and materials with them. Samples combined; M-2212 from Tomb A and M-2213 from Tomb B. Coll. 1963 by R. A. Marshall; subm. by Ray Williams, Univ. of Missouri. Comment (R.W.): date is about what was expected. Site is later than originally suspected.

\section{M-2214. Harryette site, Missouri}

$730 \pm 100$

Wood charcoal from Harryette site (23 NM 269) (36 $37^{\prime} \mathrm{N}$ Lat, $89^{\circ} 40^{\prime}$ W Long), New Madrid Co., Missouri. Sample from Feature 14, area of concentration of ash, fired clay, and bone, Sq. $1070 \mathrm{~N}, 980 \mathrm{E}$, Level 1, S.D. $7.62 \mathrm{~cm}$. Site seems to be Middle Baytown. Will help date certain ceramic type combinations in Little River lowland and their assoc. with burial patterns. Coll. 1968 by Fred Schneider; subm. by Ray Williams. Comment (R.W.): feature belonged to an early Mississippian component; date is satisfactory.

\section{Potato Patch site series, Missouri}

Wood charcoal from Potato Patch site (23 NM 217) (36 $46^{\prime}$ N Lat, $89^{\circ} 30^{\prime}$ W Long), New Madrid Co., Missouri. Site seems to be Early Baytown. Will date pre-shell-tempered ceramic site in SE Missouri. Coll. 1968 and subm. by Ray Williams.

M-2215. Potato Patch site

Sq. 1010 N, 990 E, Level 2, S.D. $43.2 \mathrm{~cm}$.

M-2216. Potato Patch site

Sq. 1010 N, 990 E, Level 5, S.D. $76.3 \mathrm{~cm}$.

General Comment (R.W.): M-2215 was expected to date Early Baytown or ca. 1500 yr earlier; M-2216 was expected to date Early Baytown or ca. 800 yr earlier.

\section{M-2217. Denton Mound site, Missouri}

A.D. 1290

$660 \pm 100$

Wood charcoal from Denton Mound site (23 Pm 549) (36 06 $6^{\prime}$ N Lat, $89^{\circ} 54^{\prime}$ W Long), Pemiscot Co., Missouri. Sample from Excavation Area I, Sq. $2490 \mathrm{~N}, 2530$ E, S.D. $43.2 \mathrm{~cm}$, large center post in probable funeral 
house. Coll. 1968 by Fred Schneider; subm. by Ray Williams. Comment (R.W.): site is thought to belong to early part of Late Mississippian Nodena phase. Date is perhaps slightly early.

\section{M-2257. Powell Bayou site, Mississippi}

$670 \pm 100$

Charcoal (Fraxinus sp.) id. by Suzanne Harris from Powell Bayou

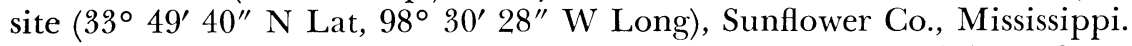
Coll. by R. A. Marshall; subm. by John Connaway, Box 367, Helena, Arkansas. Comment (R.A.M.): date fits interpretation of ceramic complex that it is primarily a Middle to late Middle Mississippian site. (J.B.G): this is site 17-0-9 of Lower Mississippi Valley Survey report (Phillips, Ford, and Griffin, 1951, Fig. 19).

\section{Salts Cave site series, Kentucky}

Human tissue from Salts Cave $\left(37^{\circ} 11^{\prime} \mathrm{N}\right.$ Lat, $86^{\circ} 03^{\prime} \mathrm{W}$ Long), Hart and Edmondson Cos., Kentucky. From male child ca. 9 yr old. Body appears to have been in flexed position on left side on floor or ledge, well-preserved except for bones of left hand and both feet. Tissue of entire left side torn away (Watson and Yarnell, 1966). Coll. and subm. by L. M. Robbins, Univ. of Kentucky, Lexington, Kentucky.

\section{M-2258. Salts Cave site}

$1960 \pm 160$ $20 \mathrm{~g}$ size.

\section{M-2259. Salts Cave site}

Two $10 \mathrm{~g}$ sizes.

General Comment (L.M.R.): satisfactory dates; they are closer than expected since tissue was taken from different parts of body. Dates agree with upper temporal range of the fecal specimens recovered from Salts Cave, i.e., $290 \pm 200$ в.c. (M-1573: R., 1968, v. 10, p. 71) and $320 \pm 140$ B.c. (M-1771: R. ibid.), and they are only slightly more recent than one of dates from Mammoth Cave $(420 \pm 60$ B.c.; Benington, Melton, and Watson, 1962). Earliest date suggests man's presence in Salts in $1190 \pm$ 150 B.c. (M-1589: R., 1968, v. 10, p. 72) for prehistoric soot from cave ceiling. Late Archaic and Early Woodland people probably occupied or visited the cave over a long period of time.

\section{M-2382. Buck site, Midden Sample No. 1, Florida A.D. 720}

$1230 \pm 120$

Charred wood, probably pine, from Buck site, Midden Sample No. 1 (8 Ok 11) $\left(30^{\circ} 24^{\prime} 00^{\prime \prime}\right.$ N Lat, $86^{\circ} 37^{\prime} 00^{\prime \prime}$ W Long), Okaloosa Co., Florida. From lowest level of midden deposit directly $S$ of burial mound, a pure deposit of Weeden Island phase. Coll. 1969 and subm. by D. S. Phelps, East Carolina Univ., Greenville, N.C. Comment (D.S.P.): dates earlier range of site occupation, Weeden Island phase, and provides minimum time limit for mound construction. It falls within estimated age. 


\section{Buck site series, Florida}

Charcoal from Buck site (8 Ok 11) $\left(30^{\circ} 24^{\prime} 00^{\prime \prime} \mathrm{N} \mathrm{Lat,} 86^{\circ} 37^{\prime} 00^{\prime \prime}\right.$ W Long), Okaloosa Co., Florida.

\section{M-2383. Buck site}

$$
1170 \pm 140
$$

Charcoal from Level 2, 20.3 to $35.6 \mathrm{~cm}$, in perimeter of mound. Coll. 1966 and subm. by Y. W. Lazarus, Temple Mound Mus., Fort Walton Beach, Florida.

\section{M-2384. Buck site}

$1470 \pm 130$

Charcoal from N sec. of Pit 7-1966, $15.2 \mathrm{~cm}$ depth. Coll. 1966 by D. W. Sharon; subm. by Y. W. Lazarus.

General Comment (Y.W.L.): should date material of Weeden Island pottery. Previous date yielded A.D. $815 \pm 150$ (FSU, AC 30) for another Weeden Island Mound with comparable material, Basin Bayou West (8 WL 13). M-2383 is more valid and satisfactory. Dates provide better estimate of time of Buck Mound people. The 3 previously tested dates for this site were unsatisfactory: $2515 \pm 140$ B.c. (GX-215), $<180 \mathrm{yr}$ B.P. (FSU-172), and n.d. (GX-154).

\section{M-2385. Grayton Beach, Florida}

$1380 \pm 130$

Charcoal from Grayton Beach $\left(30^{\circ} 20^{\prime} 10^{\prime \prime} \mathrm{N}\right.$ Lat, $86^{\circ} 12^{\prime} 08^{\prime \prime} \mathrm{W}$ Long), Walton Co., Florida. From Pit 4, $30.4 \mathrm{~cm}$ level, $\mathrm{N}$ edge of site area. Should date late Deptford or Swift Creek, but nothing more recent than very early Weeden Island I. Coll. 1961 by W. C. Lazarus; subm. by Y. W. Lazarus. Comment (Y.W.L.): date is much more valid and satisfactory than previous date, A.D. $980 \pm 130$ (FSU, AC 35; Lazarus, 1965).

\section{Bonds Village site series, Mississippi}

Charcoal from Bonds Village site (22 TU 530) (34 $32^{\prime} 30^{\prime \prime}$ N Lat, $90^{\circ} 27^{\prime} 30^{\prime \prime} \mathrm{W}$ Long), Tunica Co., Mississippi. Coll. 1968 and subm. by John Connaway.

\section{M-2163. House 2}

A.D. 1070

$880 \pm 110$

Sample 10 from SW wall trench of House 2 and Sample 11 from NW wall trench of same house from varying depths below plow zone.

\section{M-2164. House 4}

$690 \pm 100$

Sample 12 ca. $1.83 \mathrm{~m} \mathrm{~W}$ of SW corner of House 4 in area of abundant charcoal covering fired house floor. May be more of House 4 floor or different house. $.31 \mathrm{~m}$ deep.

M-2175. House 4

$780 \pm 130$

Sample 14 and 19 from S wall trench of House 4. 


\section{M-2177. House 4}

Sample 16 from $W$ wall trench of House 4.

M-2178. House 5

Samples 17 and 18 from $\mathrm{W}$ and $\mathrm{E}$ wall trenches, House 5 .

General Comment (J.C.): dates are satisfactory and agree with results from preliminary analysis of site, with generally consistent overall picture. Margin of error is large enough on all of them to afford a common date of A.D. 1160 as well as a range of $460 \mathrm{yr}$ from A.D. 920 to 1380. The 3 houses will probably fit within this period.

\section{Teoc Creek site series, Mississippi}

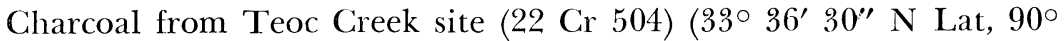
$06^{\prime} 05^{\prime \prime}$ W Long), W 1/4, SW 1/4, Sec. 9, T.20N, R.2E, Greenwood quad., Carroll Co., Mississippi. Coll. 1970 by John Connaway and Sam McGahey; subm. by John Connaway.

M-2393. Teoc Creek site

$3400 \pm 160$

Sq. 0-10E, Level 2, .305 to $.427 \mathrm{~m}$ below surface.

M-2394. Teoc Creek site

$3020 \pm 150$

Sq. 10S-10E, Level 2, .305 to $.427 \mathrm{~m}$ below surface.

\section{M-2395. Teoc Creek site}

$3650 \pm 160$

1700 B.C.

From deep midden at $\mathrm{W}$ end of profile trench, excavation area 3, ca. $2.22 \mathrm{~m}$ below surface; 3 Poverty Point objects in midden.

M-2412. Teoc Creek site

$3600 \pm 160$

1650 B.C.

Sq. 10S-10E, Level $4, .55$ to $.67 \mathrm{~m}$ below surface.

M-2413. Teoc Creek site

$3210 \pm 250$

1260 B.C.

Sq. 10S-10E, Level 5, .67 to .794 m below surface.

$3270 \pm 200$

M-2414. Teoc Creek site

1320 в.C.

Sq. 10S-10E, Level 6, .794 to $.915 \mathrm{~m}$ below surface. Small sample.

\section{M-2415. Teoc Creek site}

Sq. 0-10E, Level 3, .427 to .55 m below surface.

M-2416. Teoc Creek site

Sq. 0-10E, Level 4, .55 to $.67 \mathrm{~m}$ below surface.
$3080 \pm 150$

1130 B.c.

$3470 \pm 160$

1520 B.C. 
M-2417. Teoc Creek site

Sq. 0-10E, Level 5, .67 to $.794 \mathrm{~m}$ below surface.

General Comment (J.C.): dates seem remarkably consistent. Compared to other Poverty Point sites, they are quite acceptable. Lack of pottery in all levels of $37.2 \mathrm{~m}^{2}$ excavation area, except on the surface, indicates dates relate to pre-pottery occupation. All except earliest, M-2395, are from this excavation area. M-2395 is from an occupation zone ca. $22.2 \mathrm{~m}$ below surface of natural levee of site. Zone is probably continuous with lower levels of excavated midden on the levee backslope. Date is not much earlier than the 2 from Level 4 of the excavation.

\section{Snodgrass site series, Missouri}

Charcoal from Snodgrass site (23 BU 21-B) $\left(36^{\circ} 33^{\prime} \mathrm{N}\right.$ Lat, $90^{\circ} 33^{\prime}$ W Long), Butler Co., Missouri.

\section{M-2133. Snodgrass site}

A.D. 1480

$470 \pm 100$

Quercus, red oak group, from Str. 14, F.S. No. 106, Sq. 998.5 E, 224.1 S, D.D. 3.32 m. Coll. 1968 by J. E. Price; subm. by J. B. Griffin.

\section{M-2134. Snodgrass site}

$560 \pm 150$

Hickory (Carya sp.) from Str. 14, F.S. No. 162, Sq. 989.05 E, 225.45 S, D.D. 3.4 m. Coll. 1968 by J. E. Price; subm. by J. B. Griffin.

M-2135. Snodgrass site

$630 \pm 100$

White oak from Str. 7, Sq. 938.9 E, 231.0 S, D.D. 2.83 m. Coll. 1968 by J. E. Price; subm. by J. B. Griffin.

M-2136. Snodgrass site

$430 \pm 100$

Hickory or pecan (Carya sp.) from Str. 7, Sq. 947.0 E, 228.5 S, D.D. 3.36 m. Coll. 1968 by J. E. Price; subm. by J. B. Griffin.

\section{M-2137. Snodgrass site}

$520 \pm 100$

Charcoal, (probably Fraxinus sp.) ash, Extra Ed., Pit No. 10, NE Quad. Coll. 1968 by J. E. Price; subm. by J. B. Griffin.

M-2180. Snodgrass site

$400 \pm 100$

Quercus, white, from Str. 3, F.S. No. 324, 154.9 S, 880.3 E, D.D. 2.46 m, Post No. 5. Coll. 1967 and subm. by J. E. Price, Univ. of Michigan.

M-2181. Snodgrass site

$620 \pm 100$

Quercus, red, from Str. 3, F.S., 292, 153.5 S, 883.9 E. Coll. 1967 and subm. by J. E. Price. 
M-2182. Snodgrass site

$$
\begin{array}{r}
620 \pm 100 \\
\text { A.D. } 1330 \\
730 \pm 110 \\
\text { A.D. } 1220
\end{array}
$$

Quercus, red, from Str. 4, F.S. 299, 191.0 S, 929.0 E, D.D. $2.33 \mathrm{~m}$. Two runs made. Coll. 1967 and subm. by J. E. Price.

\section{M-2183. Snodgrass site}

$$
\text { A.D. } 1390
$$

$$
560 \pm 100
$$

Fraxinus sp. from Str. 4, 187.85 S, 929.3 E, D.D. 2.4 m. Coll. 1967 and subm. by J. E. Price.

M-2184. Snodgrass site

Carya sp. from Str. 10, 151.70 S, 99.160 E, D.D. 2.46 m. Coll. 1967 and subm. by J. E. Price.

\section{M-2185. Snodgrass site}

$$
810 \pm 110
$$

Quercus, red, from Str. 10, 150.60 S, 991.70 E, D.D. 2.5 m. Coll. 1967 and subm. by J. E. Price.

\section{M-2274. Snodgrass site}

$$
560 \pm 100
$$

Str. 15, F.S. 272, 755.8 E, 301.3 S. Coll. 1969 by J. E. Price; subm. by J. B. Griffin.

\section{M-2275. Snodgrass site}

Str. 24, S 1/2 of F.S. 1, 808.5 E, $152.05 \mathrm{~S}$ to $804.0 \mathrm{E}, 152.65 \mathrm{~S}$. Coll. 1969 by J. E. Price; subm. by J. B. Griffin.

\section{M-2430. Snodgrass site}

$$
790 \pm 100
$$

Str. 17, F.S. 89, 793.45 E, 230.56 S. Coll. 1970 by Pamela Mears and Michael Jochim; subm. by J. B. Griffin.

\section{M-2431. Snodgrass site}

Str. 17, F.S. 141, 228.4 S, 798.7 E, D.D. 2.36 m. Coll. 1970 by N. L. Hamblin and John Greene; subm. by J. B. Griffin.

\section{M-2432. Snodgrass site}

$$
660 \pm 100
$$

Str. 25, F.S. 246, 173.0 S, 779.5 E, D.D. 2.14 m. Coll. 1970 by Terence D’Altroy and C. M. S. Price; subm. by J. B. Griffin.

\section{M-2433. Snodgrass site}

$$
540 \pm 100
$$

Str. 25, F.S. 246, 173 S, 799.5 E, D.D. 2.14 m. Coll. 1970 by Gary Mono, Vida Goldstein, Terence D'Altroy, and C. M. S. Price; subm. by J. B. Griffin. 
General Comment (J.E.P. and J.B.G.): with exception of M-2431, dates are acceptable. Again, all specimens dated came from structures that burned the same day. Snodgrass site was very short lived, probably not $>10$ to $20 \mathrm{yr}$. Mean for all Snodgrass dates, except M-2431, is A.D. 1364, an acceptable date for Powers phase. Range of dates, however, indicates difficulty of establishing priority of one site over another on the basis of radiocarbon dates.

\section{Powers Fort series, Missouri}

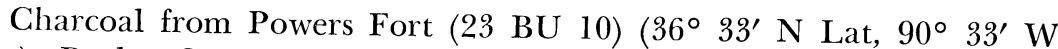
Long), Butler Co., Missouri. Coll. 1969 by John Walthall; subm. by J. B. Griffin.

\section{M-2276. Powers Fort}

Str. 1, F.S. 1, 10.6 E, 13.7 N, D.D. .305 m.

\section{M-2277. Powers Fort}

Str. 1, F.S. 44, 15.1 E, 7.5 N, D.D. .259 m.

\section{M-2278. Powers Fort}

Str. 1, F.S. 41, 16 E, 6.7 N, D.D. .259 m.

\section{M-2279. Powers Fort}

Str. 1, F.S. 40, 16.9 E, 5.6 N, D.D. .214 m. Small sample.

$540 \pm 100$

\section{A.D. 1410}

A.D. 1290

$660 \pm 100$

A.D. 1360

$590 \pm 100$

$660 \pm 200$

\section{M-2280. Powers Fort}

$650 \pm 100$

Str. 1, F.S. 47, 16.2 E, 9.2 N, D.D. .229 m.

\section{A.D. 1300}

General Comment (J.E.P.): dates are from charcoal of burned structure and should date the same. Some range in dates but they cluster better than any others run on other sites of Powers phase. Mean of Powers Fort dates is A.D. 1330, within reason for assoc. material remains.

\section{M-2434. Neil Flurry site, Missouri}

A.D. 1570

$$
380 \pm 100
$$

Charcoal from Neil Flurry site (23 BU 69) (36 $36^{\prime} \mathrm{N}$ Lat, $90^{\circ} 33^{\prime}$ W Long), Butler Co., Missouri. Str. 8, F.S. 119, 53.6 N, 82.2 E, E.D. $2.44 \mathrm{~m}$. Coll. 1970 by Timothy Hubert and Terence D'Altroy; subm. by Barbara Luedtke, Univ. of Michigan. Comment (J.E.P. and J.B.G.): date is the only one on this site and appears too recent. It does not differ greatly from some dates for other sites of the phase where $>1$ sample were run and then averaged. Date is usable but is almost certainly some 200 radiocarbon yr later than occupation.

\section{E. Great Plains}

\section{Havens site series, North Dakota}

Charcoal, small twigs, from Havens site (32 EM 1) $\left(46^{\circ} 01^{\prime} \mathrm{N}\right.$ Lat, 
$100^{\circ} 33^{\prime} \mathrm{W}$ Long), Emmons Co., North Dakota. Coll. 1967 by J. E. Sperry; subm. by W. R. Wood.

\section{M-2362. Havens site, 101-A}

From fill of Feature 164, oval pit, in NE corner of House 1.

\section{M-2363. Havens site, 101-B}

From fill of Feature 164, oval pit, in NE corner of House 1.

General Comment (W.R.W.): dates are well within apparent time range of Fort Yates phase complex of extended Missouri Valley variant and are nearly the same as one from another component of phase, Fire Heart Creek (A.D. $1230 \pm 80:$ SI-213, R., 1966, v. 8, p. 416).

\section{Paul Brave site series, North Dakota}

Charcoal, twigs, and small branches from Paul Brave site (32 Si 4) (46 $01^{\circ}$ N Lat, $100^{\circ} 35^{\prime}$ W Long), Sioux Co., North Dakota. Coll. 1955 by W. R. Wood and A. R. Woolworth; subm. by W. R. Wood.

M-2364. Paul Brave site, 918-A

$920 \pm 100$

From Excavation 2, Feature 42, fill of bell-shaped pit in front of House 2.

\section{M-2365. Paul Brave site, 918-B}

From Excavation 2, Feature 42, fill of bell-shaped pit in front of House 2.

General Comment (W.R.W.): dates are well within apparent time range of Fort Yates phase complex.

\section{Clark's Creek site series, North Dakota}

Charcoal, outer rings of an oak log, from Clark's Creek site (32 ME 1) $\left(47^{\circ} 14^{\prime} 30^{\prime \prime} \mathrm{N}\right.$ Lat, $101^{\circ} 16^{\prime} \mathrm{W}$ Long), Mercer Co., North Dakota. Should date one of most $\mathrm{N}$ components of Extended Middle Missouri variant. Coll. 1968 and subm. by W. R. Wood.

M-2366. Clark's Creek, Fl-A

In fill of Feature 1, undercut pit in Test No. 1.

\section{M-2367. Clark's Creek, Fl-B}

In fill of Feature 1, undercut pit in Test No. 1. General Comment (W.R.W.): either date is acceptable, although it seems unlikely that site was occupied long enough for both to apply.

\section{Cross Ranch site series, North Dakota}

Charcoal and wood from Cross Ranch site (32 OL 14) $\left(47^{\circ} 11^{\prime} \mathrm{N}\right.$ Lat, $101^{\circ} 00^{\prime} \mathrm{W}$ Long), Oliver Co., North Dakota. Should date one of 
most N components of Extended Middle Missouri variant. Coll. 1969 by F. A. Calabrese; subm. by W. R. Wood.

M-2368. Cross Ranch site, Sample No. 1

$420 \pm 100$

Charcoal from Feature 105, pit in floor of House 3, Level 2.

M-2369. Cross Ranch site, Sample No. 2

$590 \pm 100$

Wood from wooden brace betweer and 159, House 3.

General Comment (W.R.W.): either date is acceptable, although it seems unlikely that site was occupied long enough for both to apply.

\section{Taylor Mound site series, Kansas}

Charcoal from Taylor Mound site (14 DP 3) (39 $56^{\prime} 45^{\prime \prime} \mathrm{N}$ Lat, $95^{\circ} 15^{\prime} 30^{\prime \prime}$ W Long) NW 1/4, NW 1/4, Sec. 26, T.1S, R.39W, Doniphan Co., Kansas. Coll. and subm. by P. J. O'Brien, Kansas State Univ., Man-
hattan, Kansas.

\section{M-2343. Taylor Mound site}

$$
1940 \pm 140
$$

Feature 7, Burial 2; charcoal from cremation within cremation layer of mound.

\section{M-2344. Taylor Mound site}

$1660 \pm 140$

Feature 9; wood charcoal within cremation layer of mound ca. .61 $\mathrm{m}$ higher than cist. Cremation layer was laid down as part of ceremony covering cist, the cremation fire itself.

\section{M-2345. Taylor Mound site}

$1940 \pm 140$

Feature 9; wood charcoal from cremation layer.

$$
\text { A.D. } 10
$$

General Comment (P.J.O.): dates are expected for Valley focus Woodland.

\section{Pridey site series, Missouri}

Charcoal from Pridey site (23 PL 4) (39 $12^{\prime} \mathrm{N}$ Lat, 94 $44^{\prime} 30^{\prime \prime}$ W Long), SW $1 / 4$, SE $1 / 4$, Sec. 30, T.51N, R.34W, Platte Co., Missouri. Coll. and subm. by P. J. O'Brien.

\section{M-2346. Pridey site}

$$
660 \pm 100
$$

Charcoal from Feature 11 in House 1; from an interior support post of house.

\section{M-2347. Pridey site}

$$
880 \pm 110
$$

Wood charcoal from S wall, Sq. 10S-25W, directly above floor of house. Probably an exterior support post of House 1. 
General Comment (P.J.O.): dates are in range for Steed-Kisker focus of Middle Mississippian, but since house was probably used for $200 \mathrm{yr}, \mathrm{I}$ favor later date for house, with earlier date probably belonging to a re-used post or the like.

F. Western United States

\section{M-2348. Ruby site, Wyoming}

$$
1800 \pm 140
$$

A.D. 150

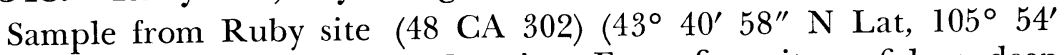
$16^{\prime \prime}$ W Long), Campbell Co., Wyoming. From fire pit ca. $6.1 \mathrm{~m}$ deep in profile cut by an arroyo. Coll. and subm. by G. C. Frison, Univ. of Wyoming, Laramie, Wyoming. Comment (G.C.F.): excellent date; it fits very well with both geologic and another carbon date from same area. $\mathrm{C}^{14}$ date, $1670 \pm 135 \mathrm{yr}$ (GX-1157: Frison, 1971), was obtained $3.05 \mathrm{~m}$ higher in same depositional sequence, giving an idea of depositional rates for alluvium in area.

\section{Glenrock Buffalo Jump series, Wyoming}

Samples from Glenrock Buffalo Jump (48 CO 304) (42 $51^{\prime} 41^{\prime \prime}$ N Lat, $105^{\circ} 55^{\prime} 58^{\prime \prime}$ W Long), Converse Co., Wyoming. Coll. and subm. by G. C. Frison.

\section{M-2349. Glenrock Buffalo Jump}

From fire hearth $1.295 \mathrm{~m}$ deep. Late Prehistoric period.

\section{M-2350. Glenrock Buffalo Jump}

$$
210 \pm 100
$$

From fire hearth $1.95 \mathrm{~m}$ deep. Late Prehistoric period.

General Comment (G.C.F.): excellent dates; they fit with other archaeologic evidence, suggesting that site was probably used until historic period, i.e., ca. A.D. 1750.

\section{M-2373. Hermanas Ruin, New Mexico}

\section{A.D. 1160}

$$
790 \pm 110
$$

Charcoal, mesquite, from Hermanas Ruin (31 $47^{\prime} \mathrm{N}$ Lat, $108^{\circ} 12^{\prime}$ W Long), Luna Co., New Mexico. Sample from Unit 490 E 500, Feature No. 1, stone lined fire pit .305 to $.55 \mathrm{~m}$ below surface. Coll. 1970 and subm. by J. E. Fitting, Case Western Reserve Univ., Cleveland, Ohio. Comment (J.E.F.): from very homogeneous site, probably occupied during middle of Mimbres development. Abundant Mimbres Bold Face ceramics and no Mimbres Polychrome. I now believe entire Polychrome period postdates A.D. 1160 .

\section{M-2374. Burro Cienaga, site No. 40, New Mexico A.D. 620}

$$
1330 \pm 130
$$

Charcoal, cedar from Burro Cienaga, Site No. $40\left(32^{\circ} 25^{\prime} \mathrm{N}\right.$ Lat, $108^{\circ} 18^{\prime}$ W Long), Grant Co., New Mexico. From burned roofing material just above floor in House No. 1. House contains Three Circles Red on White and Mimbres Bold Faced ceramics on floor. Coll. 1970 and subm. 
by J. E. Fitting. Comment (J.E.F.): earlier than dates on comparable ceramics in San Francisco drainage to $\mathrm{N}$ and suggests that Gila and Mimbres drainages will prove to be core areas for Mogollon cultural developments.

$$
\text { G. Mexico }
$$

\section{M-2249. Rancho del Escritorio, Mexico}

Shell pendant from Rancho del Escritorio, Tuxpan, Jalisco, Mexico. Heavy shell pendant in poor condition, purchased, but allegedly from burial; apparently, of Comala complex. From vendor's statements and one pottery vessel still in his possession, Comala assoc. was assumed. Coll. and subm. by Isabel Kelly, Tepepan (z.23) D.F., Mexico. Comment (I.K.): date ca. $1 / 2$ millennium earlier than expected. Whole ceramic series in Colima area may have to be pushed back in time. More likely is that rifled tomb (?) in question may have had pre-Colima use, which was not clear from limited ceramic evidence. Neither in this case nor for other dates based on shell has any adjustment for "upwelling" been applied.

\section{Cerro del Zapote series, Mexico}

Shell bracelet fragments from rifled tomb, Cerro del Zapote, Colima, Mexico. Specimen purchased from unknown vendor. Chiefly Zapote complex, but some vessels of San Miguel horizon, evidently formal part of burial furniture. Coll. and subm. by Isabel Kelly.

\section{M-2251. Cerro del Zapote}

$2320 \pm 140$

Assoc. with large number of discarded pottery vessels of both San Miguel and Zapote complexes.

\section{M-2252. Cerro del Zapote}

$2060 \pm 140$

Sample from rifled tomb, ca. $15 \mathrm{~m}$ from that which produced M-2251. Specimens purchased; assoc. with either San Miguel or Zapote complex.

General Comment (I.K.): unpub. date based on a sherd sample (Geochron: GX-1785) was reported as $3080 \pm 190$ or 1130 B.c. for Zapote complex. Latter date considered too early; as yet, no secure date exists for San Miguel horizon. Zapote and San Miguel, now considered separate complexes, may be 2 aspects of a single phase; ceramically, relationship is close.

\section{El Chanal site series, Mexico}

Charcoal from El Chanal (19 $16^{\prime} \mathrm{N}$ Lat, $103^{\circ} 41^{\prime} \mathrm{W}$ Long), Colima, Mexico. Ceramically, site does not fit into regular Colima series, because relationships seem to be with Jalisco (Autlan and Tuxcacuesco) and Michoacan. Coll. and subm. by Isabel Kelly. 


\section{M-2334. El Chanal West, Pot. Edificios Chicos}

Charcoal from Test \#1.

\section{M-2338. El Chanal, East, Cenicero} gether.

Charcoal from Test 3:5 (80 to $100 \mathrm{~cm}), 2$ pieces found close to-

General Comment (I.K.): both dates comparatively late and confirm impression that El Chanal was occupied in centuries immediately prior to the Conquest.

\section{M-2339. El Bajadero, Las Borregas, Mexico}

$$
\begin{array}{r}
1260 \pm 130 \\
\text { A.D. } 690
\end{array}
$$

Marine shell from Burial 11, El Bajadero, Las Borregas, Acatitlan (19 $06^{\prime} \mathrm{N}$ Lat, $104^{\circ} 45^{\prime} \mathrm{W}$ Long), Colima, Mexico. Coll. and subm. by Isabel Kelly. Comment (I.K.): 1st date for Armeria complex; somewhat earlier than estimated, not leaving much room for Comala and Colima complexes, which are thought to precede Armeria during 1st centuries A.D. More carbon dates are needed.

\section{M-2340. Parcela de Jacinte Avila, Mexico}

$$
\begin{array}{r}
1810 \pm 140 \\
\text { A.D. } 140
\end{array}
$$

Two fragments of marine shell, from surface of a cluster of rifled burials, Parcela de Jacinte Avila, Coahuayana Valley $\left(18^{\circ} 46^{\prime} \mathrm{N}\right.$ Lat, $103^{\circ} 40^{\prime} \mathrm{W}$ Long), Michoacan, Mexico. Coll. and subm. by Isabel Kelly. Comment (I.K.): present date should help establish some order among ill-defined complexes of $\mathrm{E}$ Colima and adjacent Michoacan. Burials accompanied by some specimens, tentatively assignable to Chanchopa phase, but certain key wares were lacking. More data are necessary.

\section{M-2341. Loma del Volantin, Alcusahue, Mexico}

Worked marine shell from rifled tomb, Loma del Volantin, Alcusahue ( $18^{\circ} 56^{\prime} \mathrm{N}$ Lat, $103^{\circ} 47^{\prime} \mathrm{W}$ Long), Colima, Mexico. Coll. and subm. by Isabel Kelly.

\section{M-2341a. Loma del Volantin}

$$
\begin{gathered}
1690 \pm 140 \\
\text { A.D. } 260 \\
2230 \pm 140 \\
280 \text { B.C. }
\end{gathered}
$$

M-2341b. Loma del Volantin

General Comment (I.K.): sherd material salvaged from tomb establishes presence of at least 2 Ortices vessels, evidently grave furniture; earlier date may refer to these. Some sherd material not yet identifiable. Nearly $550 \mathrm{yr}$ spread from one tomb > expected. Usual reservations as to utility of shell for $\mathrm{C}^{14}$ dating may apply. But, from other evidence, it is patent that tombs were used and re-used, which present dates reinforce, although somewhat extravagantly. These 2 dates emphasize perils of lumping discrete samples presumed contemporaneous. Compare 
with M-2396, based on good charcoal sample, which places a presumably Ortices deposit from Coahuayana Valley at 160 B.c.

M-2396. La Paranera, San Vicente, Mexico

Charcoal from La Paranera, San Vicente, Coahuayana Valley $\left(18^{\circ}\right.$ $44^{\prime} \mathrm{N}$ Lat, $103^{\circ} 41^{\prime} \mathrm{W}$ Long), Michoacan, Mexico. Sample from Test \#1, East pit, $340 \mathrm{~cm}$ below surface. Coll. 1970 and subm. by Isabel Kelly. Comment (I.K.): material from this test not yet tabulated but believed to be a local variety of Ortices, quite close to that recognized many years ago in Salado drainage and presumably its contemporary. Only charcoal sample so far attributable to Ortices phase; dates based on shell are M-234la and M-2341b.

\section{San Jose Mogote site series, Mexico}

Charcoal from San Jose Mogote site $\left(17^{\circ} 11^{\prime} \mathrm{N}\right.$ Lat, $96^{\circ} 48^{\prime} \mathrm{W}$ Long), Oaxaca, Mexico. Sample should date Early Formative deposit with zoned, rocker-stamped sherds and abundant worked marine shell (Flannery et al., 1967). Coll. 1966 and subm. by K. V. Flannery, Mus. Anthropol., Univ. of Michigan.

\section{M-2104. Sample OS-62, I10 and OS-62, $\quad 2670 \pm 200$ K11, Zone D3 \\ 720 B.c.}

From bottom of ashy midden layer overlying ignimbrite. Est. date: 900 to 1200 B.C. Comment (K.V.F.): expected dates for San Jose phase are 850 to 1150 B.c. Date falls within one standard deviation of range.

\section{M-2330. Area C, Feature 23}

$3280 \pm 180$

Pit is bedrock below Zo (K.V.F.): acceptable.

\section{M-2331. Area C, Sq. S33A \\ $3120 \pm 150$}

Carbonized post in bedrock below stratigraphic Zone G. Tierras Largas phase. Comment (K.V.F.): acceptable.

\section{M-2354. Area C, Sample OS-62-C/F.24}

$$
2840 \pm 150
$$

Feature 24, pit in bedrock, from flotation sample. San Jose phase. Comment (K.V.F.): acceptable.

\section{M-2355. Area C, Sq. S24A, Zone E}

$2610 \pm 150$

Sherd layer above Platform 3 and below House 5. San Jose phase. Comment (K.V.F.): too young.

\section{M-2372. Area C, Code: OS-62-C}

Charcoal from fill of Platform 5 (Zone F). Tierras Largas phase. Comment (K.V.F.): acceptable. 
Tierras Largas site series, Oaxaca, Mexico

Charcoal from Tierras Largas site $\left(17^{\circ} 05^{\prime} \mathrm{N}\right.$ Lat, $96^{\circ} 46^{\prime} \mathrm{W}$ Long), Oaxaca, Mexico. Coll. 1969 and subm. by K. V. Flannery.

\section{M-2351. Sq. 1995, Sample 1}

$2960 \pm 150$

Tierras Largas phase.

M-2352. Sq. 1005, from below metate No. 2

Charcoal. Tierras Largas phase.

\section{M-2353. Feature 116}

$80 \mathrm{~cm}$ below top of bedrock. Tierras Largas phase.

1080 B.c.

General Comment (K.V.F.): expected date for Tierras Largas phase is 1400 to 1150 B.c. Dates are within one standard deviation of above range.

\section{San Sebastian Abasolo site series, Oaxaca, Mexico}

Charcoal from San Sebastian Abasolo (16 $56^{\prime} \mathrm{N}$ Lat, $96^{\circ} 32^{\prime} \mathrm{W}$ Long), Oaxaca, Mexico. Coll. 1969 and subm. by K. V. Flannery.

\section{M-2358. Test A, Sq. E 13, Feature 3}

$2600 \pm 150$

From Pre-Columbian well. Comment (K.V.F.): well is San Jose phase (1150 to 850 B.c.). Date is therefore too recent. Sample was from below ground water level in bottom of well and may be contaminated.

\section{M-2357. Test A, Sq. D 13, Zone D2}

$2680 \pm 200$

Depth of $220 \mathrm{~cm}$. Comment (K.V.F.): expected date is 900 B.c. Date is within one standard deviation.

\section{M-2360. Test B}

$2330 \pm 180$

Depth of $120 \mathrm{~cm}$ below surface. Monte Alban I. Comment (K.V.F.): perfect.

$$
2730 \pm 150
$$

\section{M-2371. Feature 3-fill}

Charcoal from San Jose phase well. Comment (K.V.F.): date is within one standard deviation of expected date of 900 B.C.

\section{Barrio del Rosario Huitzo, Mexico}

Charcoal from Barrio del Rosario Huitzo (17 $17^{\circ} 17^{\prime} \mathrm{N}$ Lat, $96^{\circ} 54^{\prime}$ W Long), Oaxaca, Mexico. Coll. 1967 and subm. by K. V. Flannery.

\section{M-2102. Sample B46A, Zone F3}

$$
\begin{aligned}
& 2800 \pm 150 \\
& 850 \text { B.C. }
\end{aligned}
$$

Charcoal from burned post embedded in sterile soil at base of stratigraphic column, $7.5 \mathrm{~m}$ below surface. Should date Early Formative. Geochron date from same log: $1400 \pm 140$ B.c. (Flannery et al., 1967). Comment (K.V.F.): acceptable date. 
M-2386. Area B46C ( V-W site), Zone D2

Midden charcoal. Comment (K.V.F.): within one standard deviation of expected date ca. 600 в.c.

M-2387. Area B46A (control), House 7

$2960 \pm 150$

Charcoal (fuel) from floor of House 7, Guadalupe phase. Comment (K.V.F.): expected date is ca. 850 B.c.; sample is roughly within one standard deviation of it.

\section{H. Africa, Europe, Near East, and Asia}

\section{Ita Yemoo site series, Nigeria}

Charcoal from Ita Yemoo site $\left(07^{\circ} 28^{\prime} \mathrm{N}\right.$ Lat, $04^{\circ} 32^{\prime} \mathrm{E}$ Long), Ife, Nigeria. Coll. and subm. by Frank Willett, Northwestern Univ., Evanston, Illinois.

\section{M-2117. Ita Yemoo 1397}

A.D. 1470

$480 \pm 100$

From Layers 4 and 5, very small trench cutting entirely sealed by potsherd pavement No. 1 and overlain by stone pavement No. 15. Sample from $3.81 \mathrm{~m}$ to $.854 \mathrm{~m}$ below top pavement and immediately below stone pavement. BM-261, directly above, dated A.D. $960 \pm 130$ (R., 1969, v. 11, p. 291).

\section{M-2119. Ita Yemoo Unnumbered, 1072 and 1187 \\ A.D. 1150 \\ $800 \pm 200$}

Combined sample from Layer 2 over pavement and beside terra cotta. Unnumbered: Sq. 7D, Layer 2. Corresponds to BM-262: A.D. $1060 \pm 130$ (R., 1969, v. 11, p. 291); IY 1072, Sq. 4E, Layer 2 of reddishbrown earth.

\section{M-2120. Ita Yemoo 1066}

A.D. 1350

$600 \pm 100$

Layer 13A, Trench XIII. Two sections: lower part of fill of Feature 9, deep pit outside town wall, $2.31 \mathrm{~m}$ below surface. Upper: deep pit, obscure but cut by Feature 10, pit dug from old land surface. Trench XIII sectioned town wall and revealed pavement, various pits, and old wells beneath and beyond wall.

\section{M-2121. Ita Yemoo 863}

$1100 \pm 120$

Trench XIII, Feature 1, pit filling of Layer 7A, $1.98 \mathrm{~m}$ below surface. Old well excavated to $4.6 \mathrm{~m}$ below top of natural bedrock, dug from old land surface; buried below material cleaned out of town ditch. Terminus post quem for building town wall.

General Comment (F.W.): pottery from well (M-2121) is oldest so far excavated for Ita Yemoo; older rubbish must have filled it for it is stratigraphically later than BM-259: A.D. $1160 \pm 130$ (R., 1969, v. 11, p. 291) and M-2119 which corresponds well with BM-262 from same 
layer. M-2117 comes from below a pavement which corresponds stratigraphically to that below M-2119 and BM-262; it exactly overlays BM261 in Layer 11 and must be contaminated, probably from organic material accumulated in a deep hollow in overlying pavement. Cire perdue brasscastings were found and appear to have come from a corresponding situation and to be from 11th and 12th centuries, when nearly life-size naturalistic terra cotta sculptures were being made.

\section{Orun Oba Ado site series, Nigeria}

Charcoal from Orun Oba Ado site $\left(07^{\circ} 28^{\prime} \mathrm{N}\right.$ Lat, $04^{\circ} 32^{\prime} \mathrm{E}$ Long), Ife, Nigeria. Site of burial of heads of kings of Benin in Ife. Coll. and subm. by Frank Willett.

\section{M-2114. Pit III sample}

$1150 \pm 120$

Depth of $.764 \mathrm{~m}$ in Pit III, Sq. C3 adjacent to D in dark brown earth Layer 4; large, irregular, non-gravel pit.

\section{M-2115. Pit V sample}

$1150 \pm 120$

Combination of small samples 112, 113, 114, 138, 142, 152 from 2.44 to $2.75 \mathrm{~m}$ below surface.

\section{M-2116. Pit VI sample}

$$
1010 \pm 150
$$

Combination of very small charcoal samples 32, 83, 96, 117, 118, $119,123,139,144,149$ at depths of $.812 \mathrm{~m}$ to $2.14 \mathrm{~m}$.

General Comment (F.W.): taken together, these dates confirm that site of Ife itself was occupied during latter part of 1st millennium A.D., earlier than some scholars would expect and earlier than generally accepted date for beginning of Yoruba Dynasty in Benin. Pottery from Orun Oba Ado appears less evolved than main series from Ita Yemoo; that Orun Oba Ado dates should mostly precede those from Ita Yemoo is satisfactory (cf. BM-265: A.D. $560 \pm 130$ and BM-264: A.D. $990 \pm 130$ from Orun Oba Ado: R., 1969, v. 11, p. 292).

\section{M-1325. Mound 1, Leki Male, Poland \\ Wood from Mound 1 at Leki Male $\left(52^{\circ} 11^{\prime} \mathrm{N}\right.$ Lat, $16^{\circ} 33^{\prime}$ E Long),}

$3900 \pm 150$ Koscian dist., Poland. Piece of wood from Burial A in Mound (Barrow) 1, 3rd layer of stone construction, Bronze age Unetice culture (Kowianska-Piaszykowa and Kurnatowski, 1954). Coll. 1953 by Mieczyslawa Kowianska-Piaszykowa; subm. by J. B. Griffin. Comment (S.R. Milisauskas): with relative chronology, excavators date mound ca. 1700 to 1500 в.c. $\mathrm{C}^{14}$ date is earlier than expected for Early Bronze age Unetice culture in Poland. Same mound dated by Groningen, GrN-5037 at 3605 \pm 40 (Bakker et al., 1969, p. 1516). Considering standard deviation, however, date is probably acceptable. 


\section{Olszanica site series, Poland}

Charcoal from Olszanica site $\left(50^{\circ} 06^{\prime} \mathrm{N}\right.$ Lat, $18^{\circ} 50^{\prime} \mathrm{E}$ Long) near Stalinograd and Gliwice, S Poland. Coll. 1968 by Janusz Kruk; subm. by Sarunas Milisauskas, New York State Univ. at Buffalo.

\section{M-2165. Olszanica}

$6150 \pm 210$

Charcoal from Feature No. 1, 57 to $59 \mathrm{~cm}$ depth. Most sherds in feature belong to Zeliezorska phase (III) of Linear culture in Little Poland.

\section{M-2314. Olszanica $\mathbf{D}_{1}$}

Charcoal from Feature \#12/S, Area $\mathrm{D}_{1}, 70$ to $90 \mathrm{~cm}$ depth. Feature designated as Danubian I phase of Linear culture.

\section{M-2320. Olszanica $\mathbf{D}_{1}$}

Charcoal from Feature 1/1969, Level 19, 100 to $120 \mathrm{~cm}$ depth. Cultural assocs. indicate Danubian I phase of Linear culture.

General Comment (S.M.): M-2165 date is good. Earliest expected date for M-2314 was ca. 6400 or 6450 B.P. M-2320 dates Feature 1/1969 near end of Linear culture.

\section{Iwanowice site series, Poland}

Charcoal from Iwanowice site $\left(50^{\circ} 13^{\prime} \mathrm{N}\right.$ Lat, $19^{\circ} 58^{\prime} \mathrm{E}$ Long $)$ near Krakov, S Poland. Coll. 1968 by Jan Machnik; subm. by Sarunas Milisauskas.

M-2166. Iwanowice

Charcoal from Feature 21, 40 to $60 \mathrm{~cm}$ depth. Feature id. as Baden culture, Late Neolithic.

\section{M-2167. Iwanowice}

Charcoal from Feature $30 \mathrm{a}, 80$ to $90 \mathrm{~cm}$ of site. Feature contained Mierzanowice culture (Early Bronze age) sherds and some Lusatian cultural sherds.

M-2168. Iwanowice
Charcoal from Feature

$3720 \pm 180$ 1770 B.c. as Mierzanowice culture, Early Bronze age in Little Poland.

\section{M-2169. Iwanowice}

Charcoal from Feature 61,70 to $80 \mathrm{~cm}$. Feature id. as Trzciniec culture, Bronze age II in Little Poland. 
M-2325. Iwanowice

Charcoal from Feature $62 \mathrm{~S}, 230$ to $250 \mathrm{~cm}$. Feature designated as Mierzanowice culture, Early Bronze age.

M-2327. Iwanowice

$3440 \pm 170$

Charcoal from Feature 61,110 to $120 \mathrm{~cm}$. Feature designated as Mierzanowice culture, Early Bronze age.

M-2328. Iwanowice

$3800 \pm 170$

1850 в.c.

Charcoal from Feature 62S, 190 to $200 \mathrm{~cm}$. Feature designated as Mierzanowice culture, Early Bronze age.

General Comment (S.M.): all dates in series are satisfactory except M-2327. Expected date for M-2327 was 3700 B.P. M-2328 is relatively early for Mierzanowice culture in S Poland. M-2167 represents acceptable date for early phase of Lusatian culture.

\section{Niedzwiedz I site series, Poland}

Charcoal from Niedzwiedz I site $\left(50^{\circ} 14^{\prime} \mathrm{N}\right.$ Lat, $20^{\circ} 08^{\prime} \mathrm{E}$ Long), near Krakov, S Poland. Coll. by Barbara Burchard; subm. by Sarunas Milisauskas.

\section{2321. Niedzwiedz I site}

Charcoal from Feature $71 \mathrm{E}, 140 \mathrm{~cm}$. Samples should date Funnel Beaker culture.

M-2322. Niedzwiedz I site

$4600 \pm 190$

Charcoal from Feature $68 \mathrm{E}, 180$ to $182 \mathrm{~cm}$. Sample should date Funnel Beaker culture.

M-2323. Niedzwiedz I site

$4640 \pm 190$ 2690 B.c.

Charcoal from Feature 62A, $240 \mathrm{~cm}$. Sample should date Funnel Beaker culture.

General Comment (S.M.): all 3 dates are satisfactory.

\section{M-2324. Raciborz-Obora, Poland}

$$
1080 \pm 120
$$

Charcoal from Raciborz-Obora site $\left(50^{\circ} 06^{\prime} \mathrm{N}\right.$ Lat, $18^{\circ} 10^{\prime} \mathrm{E}$ Long), near Raciborz, S Poland, from Mound 6A, 40 to $45 \mathrm{~cm}$. Coll. by Elizbieta Dabrokiska; subm. by Sarunas Milisauskas. Comment (S.M.): early Slav cemetery containing cremated burials. Chronology based on ceramics dates cemetery from 6 th to 9 th century A.D. Date is acceptable.

M-2455. Lupl anica-Visoko Brdo, Yugoslavia

$5600 \pm 200$

3650 B.B.

Wood charcoal from Lupl anica-Visoko Brdo $\left(45^{\circ} 20^{\prime} \mathrm{N}\right.$ Lat, $18^{\circ}$

E Long), near Derventa, Bosnia, Yugoslavia, from $1.3 \mathrm{~m}$ below ground 
surface in Unit II/1970, the lower building level of a Late Neolithic structure in SW part of site. Coll. 1970 by H. M. Wobst; subm. by J. B. Griffin. Comment (H.M.W.): excavation of this multicomponent site is still in progress. Date is satisfactory pending further excavation.

\section{Turlu series, Turkey}

Ashes from Turlu $\left(37^{\circ} 03^{\prime} \mathrm{N}\right.$ Lat, $37^{\circ} 45^{\prime} \mathrm{E}$ Long), S Turkey. Coll. 1965 and subm. by Jean Perrot, French Consulate General, POB 182, Jerusalem.

\section{M-1844, M-1844a. Turlu}

M-1844 is ashes from ashen layer at end of Halaf period: 4500 to 4000 в.c. M-1844a is ashes from hearth at end of Ubaid period: 4250 to 3750 B.C.

General Comment (J.B.G.): combined by accident. Date substantiates Perrot's expectations.

\section{Tepe Farukhabad site series, Iran}

Wood charcoal from Tepe Farukhabad site $\left(37^{\circ} 35^{\prime} 06^{\prime \prime} \mathrm{N}\right.$ Lat, $47^{\circ}$ 13' 30" E Long), Deh Luran Plain, Kermanshah Prov., Iran. Coll. 1968 by R. C. Gibbs and Robert Betteral; subm. by H. T. Wright, Mus. Anthropol., Univ. of Michigan.

\section{M-2151. Tepe Farukhabad}

$3990 \pm 180$

Wood charcoal from Excavation B, Layer 23, F \#15; lower layer, refuse-filled granary. Late Jemdet Nasr phase with expected date, 2400 B.C.

\section{M-2152. Tepe Farukhabad}

$4460 \pm 190$

Wood charcoal from Excavation A, Layer 21, F \#24; hearth designated as Late Uruk phase.

\section{M-2153. Tepe Farukhabad}

Wood charcoal from Excavation B, Layer 45, F \#41, fill from Rms. $\mathrm{A}, \mathrm{C}$, and $\mathrm{G}$.

\section{M-2419. Tepe Farukhabad}

$3800 \pm 160$

Charcoal, probably tamarisk, from Excavation B, Layer 23, F \#15, lower layer which was refuse-filled granary. M-2419 from same sample as M-2151, and was to provide a check on M-2151 which is younger than expected.

General Comment (H.T.W.): further Jemdet Nasr samples must be run before M-2151 and M-2419 can be evaluated. M-2152 compares well with later Middle Uruk at Nippur, S Iraq, dated 2722 в.c. (P-530, R., 1963 , v. 5, p. 85). M-2153 compares well with samples of preceding Bayat 
phase at nearby Tepe Sabz which averaged 4000 B.c. (Hole, Flannery, and Neely, 1969).

\section{Qasr al Hayr site series, Syria}

Wood charcoal and wood from Qasr al Hayr $\left(35^{\circ} 05^{\prime} 30^{\prime \prime} \mathrm{N}\right.$ Lat, $34^{\circ} 04^{\prime} 20^{\prime \prime}$ E Long), Syria. Site is divided by standing monuments into a "Small Enclosure," a single huge building, and a "Large Enclosure," a city with several buildings.

\section{M-2155. Qasr al Hayr}

$1220 \pm 120$

Wood charcoal from L.E. $2 \mathrm{~K}, 30 \mathrm{~cm}$ above floor, probably roof beam from Building A in Large Enclosure. Archaeologically, it is 2 generations later than construction assoc. with M-2156. Coll. 1966 by William Trousdale; subm. by Oleg Grabar, Univ. of Michigan and Harvard Univ., Cambridge, Mass.

\section{M-2156. Qasr al Hayr}

$1400 \pm 130$

Wooden part of beam in original masonry of outside wall of SE corner of palace in Small Enclosure. Coll. 1966 by Robert Hamilton; subm. by Oleg Grabar.

\section{M-2272. Qasr al Hayr}

$920 \pm 120$

Wood fragments from Building 14 C-D of Large Enclosure, unfinished court of 1st period, used as a dump. Coll. 1969 by William Trousdale; subm. by Oleg Grabar.

\section{M-2273. Qasr al Hayr}

$1080 \pm 120$

Wood fragments from building at $\mathrm{N}$ gate of Large Enclosure. No clear stratification. Coll. 1969 by Renata Holod-Tretiak; subm. by Oleg Grabar.

\section{M-2456. Qasr al Hayr}

$610 \pm 120$

Wood fragments from $\mathrm{E}$ gate building, Level 3 of Large Enclosure. Probably roofing poles from fill ca. $10 \mathrm{~cm}$ over plastered floor marking latest observable occupation of Phase 3 in room of $\mathrm{E}$ gate building. Small sample. Coll. 1970 by R. McC. Adams; subm. by Oleg Grabar.

General Comment (O.G.): dates are satisfactory. M-2273 was expected to date before A.D. 1300 but later than A.D. 1000. Since it was part of an object, wood could have been much earlier than occupation level where it was found. M-2456 comes from latest level of 2nd major phase of occupation and shortly before abandonment of site; literary and ceramic evidence indicate abandonment ca. A.D. 1320.

\section{Pulur site series, Turkey}

Charcoal from Pulur site (ca. $38^{\circ} 30^{\prime} \mathrm{N}$ Lat, $38^{\circ} 55^{\prime} \mathrm{E}$ Long), Turkey. Chalcolithic prehistoric settlement near Euphrates R.; connec- 
tions with Karaz-Aras-Malatya. Khirbet Kerak culture. Coll. 1968 by H. Z. Kosay; subm. by Robert Whallon, Jr., Mus. Anthropol., Univ. of Michigan.

\section{M-2170. Pulur site}

Excavation K, 37.9 D 29. Fifth level from top of hill.

\section{M-2171. Pulur site}

Hearth, Level 6, ca. 3000 to 3500 B.C.

\section{M-2172. Pulur site}

Level $8.5 \mathrm{~m}$ deep. Chalcolithic, ca. 4000 B.c.

\section{M-2173. Pulur site \\ Operation A I. Level 9, $7 \mathrm{~m}$ deep. Chalcolithic, ca. 4000 в.c.}

General Comment (R.W.): dates seem much more recent than might be expected, not surprising for locally persistent and developed "Khirbet Kerak" or East Anatolian Early Bronze age culture; dates are from upper levels of site which has 11 major occupational levels going to 11 $\mathrm{m}$ depth before reaching virgin soil.

\section{M-2286. Chinese Bodhissatva}

$150 \pm 100$

Wood, probably teak, from Chinese Bodhissatva statue, location known only as China. Statue is in style of early T'ang period. Dates of the few wooden samples of this style are questionable. Purchased in China in late 1940's by a private collector; now on loan to Mus. of Art, Univ. of Michigan. Subm. by Mus. of Art, Univ. of Michigan, Mrs. W. M. Spink, Ass't. Curator. Comment (W.M.S.): we must conclude that this Bodhissatva is an archaistic work in early T'ang tradition. Date is satisfactory.

\section{REFERENCES}

Bakker, J. A., Vogel, J. C., and Wrislanski, T., 1969, TRB and other $\mathrm{C}^{14}$ dates from Poland, Part A: Helinium, v. 9, p. 3-27.

Barker, Harold, Burleigh, Richard, and Meeks, Nigel, 1969, British Museum natural radiocarbon measurements VI: Radiocarbon, v. 11, p. 278-94.

Benington, F., Melton, C., and Watson, P. J., 1962, Carbon dating Prehistoric soot from Salts Cave, Kentucky: Am. Antiquity, v. 28, p. 238-41.

Buckley, J. D. and Willis, E. H., 1970, Isotopes' radiocarbon measurements VIII: Radiocarbon, v. 12, p. 87-129. Crane, H. R., 1961, The $\mathrm{CO}_{2}-\mathrm{CS}_{2}$ Geiger Counter: Rev. Sci. Instruments, v. 32, p.
953-962.

Crane, H. R. and Griffin, J. B., 1961, University of Michigan radiocarbon dates VI: Radiocarbon, v. 3, p. 105-125. p. $61-114$. 12, p. $161-180$.

Flannery, K. V., Kirkby, A. V. T., Kirkby, M. J., and Williams, A. W., Jr., 1967, Farming systems and political growth in ancient Oaxaca: Science, v. 158, no. 3800 , p. 445-54. 
Frison, G. C., 1971, The Buffalo Pound in Northwestern Plains Prehistory: Am. Antiquity, v. 36 , p. 77-91.

Goldthwait, R. P., Dreimanis, Aleksis, Forsyth, J. L., Karrow, P. F., and White, G. W., 1965, Pleistocene deposits of the Erie Lobe, in: Wright, H. E. and Frey, D. G., (eds.) The Quaternary of the United States, Princeton, N.J., Princeton Univ. Press, p. 85-97.

Hole, F., Flannery, K. V., and Neely, J. A., 1969, Prehistoric human ecology of the Deh Luran Plain: Univ. of Michigan Mus. of Anthropol., mem. no. 1.

Kowianska-Piaszykowa, M. and Kurnatowski, S., 1954, Kurhan kultury unietyckiej w Lekach Malych pow Koscian (Mound of Unetice Culture at Leki Male, Koscian District): Fontes Archaeol. Posnanienses, v. 4, p. 43-75.

Lazarus, W. C., 1965, Alligator Lake, a ceramic horizon site on the northwest Florida coast: Florida Anthropologist, v. 18, no. 2, p. 83-124.

Libby, W. F., 1954, Chicago radiocarbon dates IV: Science, v. 119, p. 135-140.

Long, Austin and Mielke, J. E., 1966, Smithsonian Institution radiocarbon measurements III: Radiocarbon, v. 8, p. 413-422.

Moselage, J., 1965, A house pattern from the Dupree site: Arkansas Archaeologist, v. 6 , no. 1 .

Phillips, P., Ford, J. A., and Griffin, J. B., 1951, Archaeological survey in the Lower Mississippi alluvial valley, 1940-1947: Papers of the Peabody Mus. of Am. Archaeol. and Ethnol., Harvard Univ., v. 25.

Smith, C. P., 1967, Current research in the Southeast, Missouri: Beckwith's Fort: Southeastern archaeological conf. Newsletter, v. 11, no. 2, p. 15-16.

Stuckenrath, R., Jr., 1963, University of Pennsylvania radiocarbon dates VI: Radiocarbon, v. 5, p. 82-103.

Stuiver, Minze and Suess, H. E., 1966, On the relationship between radiocarbon dates and true sample ages: Radiocarbon, v. 8, p. 534-540.

Thomas, C., 1894, Report on the mound explorations of the Bureau of Ethnology: Bur. Am. Ethnol., 12th ann. rept., p. 185-88.

Watson, P. J. and Yarnell, R. A., 1966, Archaeological and paleoethnobotanical investigations in Salts Cave, Mammoth Cave National Park, Kentucky: Am. Antiquity, v. 31 , p. $842-49$.

Webb, W. S., 1940, The Wright Mounds: Sites 6 and 7, Montgomery County, Kentucky: Univ. of Kentucky Repts. in Anthropol. and Archaeol., v. 5, no. 1.

1941, The Morgan Stone Mound: Site 15, Bath County, Kentucky: Univ. of Kentucky Repts. in Anthropol. and Archaeol., v. 5, no. 3.

1943, The Crigler Mounds: Sites Be 20 and Be 27 and the Hartman Mound: Site Be 32, Boone County, Kentucky: Univ. of Kentucky Repts. in Anthropol. and Archaeol., v. 5, no. 6, p. 505-579.

Webb, W. S. and Elliot, J. B., 1942, The Robbins Mounds: Sites Be 3 and Be 14, Boone County, Kentucky: Univ. of Kentucky Repts. in Anthropol. and Archacol,, v. 5 , no. 5 .

Webb, W. S. and Snow, C. E., 1959, The Dover Mound: Univ. of Kentucky Press.

Wilkins, E. S., Jr., 1962, A preliminary report on the Harlan Mill Steatite Quarry: Archaeol. Soc. Delaware Bull., no. 2, n.s., p. 1-22.

Williams, S., 1954, An archaeological study of the Mississippian culture in southeast Missouri: Ph.D. dissert., Yale Univ.

Wright, J. V., 1966, The Ontario Iroquois tradition: Natl. Mus. Canada, Bull. 210, Anthropol. ser. no. 75 . 